\title{
Integrated Current Sensor using Giant Magneto Resistive (GMR) Field Detector for Planar Power Module
}

by

\begin{abstract}
Woochan Kim
Thesis submitted to the faculty of the Virginia Polytechnic Institute and State University in partial fulfillment of the requirements for the degree of
\end{abstract}

Master of Science

In

Electrical Engineering

Khai D.T. Ngo (Chair)

Guo-Quan Lu

Louis Guido

November 16, 2012

Blacksburg, Virginia

Keywords: GMR, field detector, planar power module, signal-conditioning circuit, over-current protection, integrated gate driver circuit 


\title{
Integrated Current Sensor using Giant Magneto Resistive (GMR) Field Detector for Planar Power Module \\ Woochan Kim
}

\begin{abstract}
Conventional wire bond power modules have limited application for high-current operation, mainly because of their poor thermal management capability. Planar power modules have excellent thermal management capability and lower parasitic inductance, which means that the planar packaging method is desirable for high-power applications. For these reasons, a planar power module for an automotive motor drive system was developed, and a gate-driver circuit with an over-current protection was planned to integrate into the module. This thesis discusses a current-sensing method for the planar module, and the integrated gate driver circuit with an over-current protection. After reviewing several current-sensing methods, it becomes clear that most popular current-sensing methods, such as the Hall-Effect sensor, the current transformer, the Shunt resistor, and Rogowski coils, exhibit limitations for the planar module integration. For these reasons, a giant magneto resistive (GMR) magnetic-field detector was chosen as a current-sensing method.

The GMR sensor utilizes the characteristics of the giant magneto resistive (GMR) effect in that it changes its resistance when it is exposed to the magnetic-flux. Because the GMR resistor can be fabricated at the wafer level, a packaged GMR sensor is very compact when compared with conventional current sensors. In addition, the sensor detects magnetic-fields, which does not require direct contact to the current-carrying conductor, and the bandwidth of the sensor can be up to $1 \mathrm{MHz}$, which is wide enough for the switching frequencies of most of motor drive applications. However, there are some limiting factors that need to be considered for accurate current measurement:
\end{abstract}


- Operating temperature

- Magnetic-flux density seen by a GMR resistor

- Measurement noise

If the GMR sensor is integrated into the power module, the ambient temperature of the sensor will be highly influenced by the junction temperature of the power devices. Having a consistent measurement for varying temperature is important for module-integrated current sensors. An experiment was performed to see the temperature characteristics of a GMR sensor. The measurement error caused by temperature variation was quantified by measurement conditions. This thesis also proposes an active temperature error compensation method for the best use of the GMR sensor.

The wide current trace of the planar power module helps to reduce the electrical/thermal resistance, but it hinders having a strong and constant magnetic-field-density seen by the GMR sensor. In addition, the eddy-current effect will change the distribution of the current density and the magnetic-flux-density. These changes directly influence the accurate measurement of the GMR sensor. Therefore, analyzing the magnetic-flux distribution in the planar power module is critical for integrating the GMR sensor.

A GMR sensor is very sensitive to noise, especially when it is sensing current flowing in a wide trace and exposed to external fields, neither of which can be avoided for the operation of power modules. Post-signal processing is required, and the signal-conditioning circuit was designed to attenuate noise. The signal-conditioning circuit was designed using an instrumentation amplifier, and the circuit attenuated most of the noise that hindered accurate measurement. The over-current protection circuit along with the gate driver circuit was designed, and the concept was verified by experiments. The main achievements of this study can be summarized as:

- Characterization of conventional current-sensing methods

- Temperature characterization of the GMR resistor 
- Magnetic-flux distribution of the planar power module

- Design of the signal-conditioning circuit and over-current protection circuit 


\section{Acknowledgements}

I would like to take this opportunity to express my great appreciation to my advisor, Dr. Khai Ngo. He is the person who introduced me to the area of packaging, which gave me the motivation to get involved in CPES. I thank him for his kind guidance of my research, so I could learn how to think more deeply to overcome obstacles, and thus could ask the right question to solve the problem. His brilliant ideas and extensive knowledge showed me a way to solutions when I was puzzled. Without his guidance and persistent help, the work for my master's study would not have been possible.

I would also like to express the deep appreciation to my committee member Dr. Guo-Quan Lu, for his valuable advice and guidance to the future research.

I am also grateful to my committee members Dr. Dong S. Ha and Dr. Louis Guido. I owe them a special note of appreciation for their thoughtful guidance and constant encouragement.

I also would like to special thanks to CPES faculties. They always provided assistance to me and all CPES students behind the scenes. Without them, this extensive research facility would not be maintained.

Finally, I would like to acknowledge my debt of gratitude to my colleagues at CPES. Their specialized knowledge inspired me a great deal, and they always happily discussed with the problems I had. I may not able to list all the names, but some notable people include: Zhemin Zhang, Yin Wang, Di Xu, Tao Tao, Mudassar Khatib, Jiang Li, Pei-Hsin Liu, Wei Zhang, and Jason Zheng.

I also have deepest gratitude to my fiancée Hyunsoo Koh for her constant support and advice as a colleague. Finally, I would like to special thanks to my parents and sister, for their love, support, and prayers for me. 


\section{TABLE OF CONTENTS}

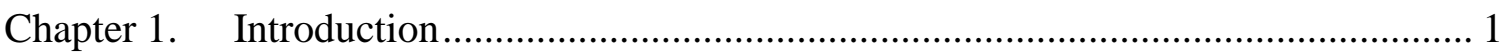

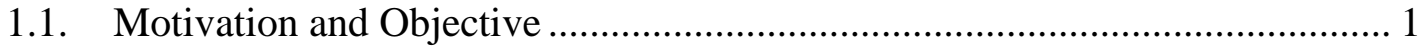

1.2. Review of Current-sensing Methods ............................................................ 3

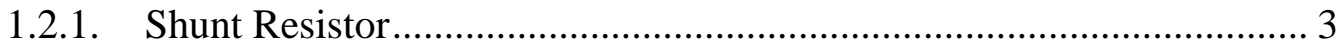

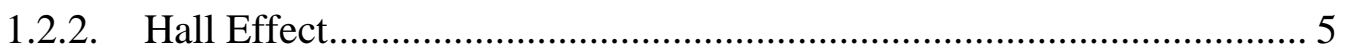

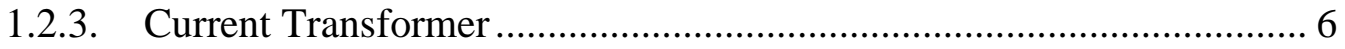

1.2.4. Anisotropic Magneto Resistive (AMR) Sensor ..................................... 6

1.2.5. Giant Magneto Resistive (GMR) Sensor ........................................... 9

1.2.6. Summary of the Current-sensing Methods .......................................... 10

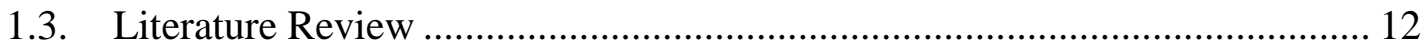

1.3.1. Output voltage of the GMR sensor by sensing location ...................... 12

1.3.2. Influence of the Frequency to the GMR sensor................................... 14

1.3.3. Power module integrated GMR sensor................................................. 14

1.4. Significance and Objective of This Study …….............................................. 15

Chapter 2. Principles and Characteristics of GMR Sensor............................................ 18

2.1. Basic Operating Principles of the GMR Sensor ........................................... 18

2.1.1. The Physics of the GMR Resistor ................................................... 18

2.1.2. Configuration as Current Sensor ……………………………….... 20

2.2. Thermal Characteristics .......................................................................... 21

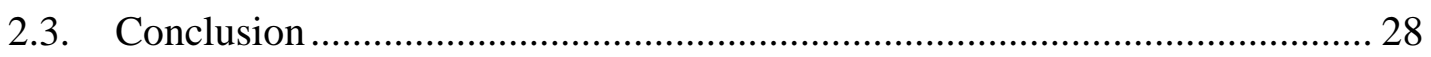

Chapter 3. Magnetic-flux Distribution of the Planar Power Module ............................ 29

3.1. Structure of the Planar Power Module …………………………………....... 29

3.2. Simulation Model for Finite Element Analysis .............................................. 31

3.3. Analysis of the Magnetic-flux Distribution.................................................... 32

3.4. Influence of the Frequency and Amplitude to Magnetic-field Distribution... 34

3.4.1. The harmonic frequency analysis of the magnetic-flux density.......... 34

3.4.2. The influence of the Eddy effect to the measurement ......................... 37

3.4.3. The influence of the Alignment of the Sensor..................................... 38

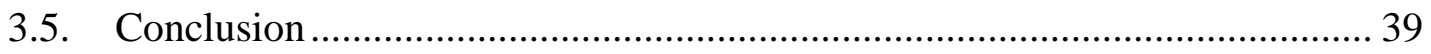


Chapter 4. Integration of the GMR Sensor into the Planar Power Module................. 41

4.1. The Test Setup of the GMR Sensor - Double Pulse Tester ........................... 41

4.2. Preliminary Measurement of the GMR Sensor ......................................... 45

4.3. Design of the Signal-conditioning Circuit ................................................. 46

4.4. Design of the Over-current Protection Circuit ............................................ 50

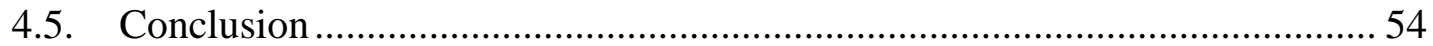

Chapter 5. Conclusions and Future Work ...................................................................... 55

5.1. Main Contributions and Conclusions ...................................................... 55

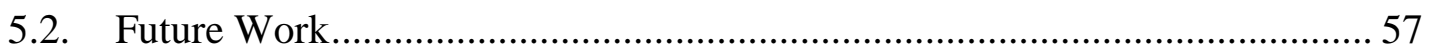

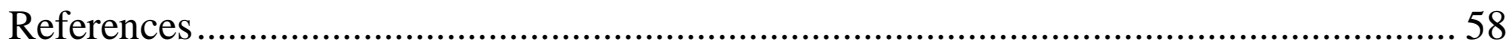

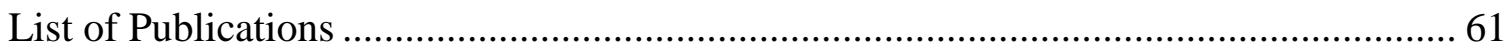


Figure 1. (a) Lay-up of a planar module; (b) planar power module after assembly.......... 1

Figure 2. (a) GMR sensing element in die form; (b) schematic of the GMR sensor in a standard IC package [32].

Figure 3. (a) The schematic of the Shunt resistor. (b) Shunt resistor in a commercial power module (Siemens SINAMICS G120).

Figure 4. (a) The operating principle of the Hall Element. (b) The Hall Effect sensor with toroidal flux concentrator.

Figure 5. (a) Operating principles of the transformer. (b) Illustration of the current transformer in use.

Figure 6. Operating principles of the AMR sensor. (a) Without external magnetic-flux. (b) With external magnetic-flux

Figure 7. Resistance change by the angle between current and magnetization vector...... 8 Figure 8. The structure of the AMR current sensor. (a) Fabrication of the AMR resistor with Barber Poles. (b) The AMR current sensor in the Wheatstone bridge structure. ....... 9 Figure 9. Relationship between GMR resistor and applied magnetic field. ..................... 9

Figure 10. Size comparison of the commercial current sensors. ................................... 11

Figure 11. Literature review 1: The output voltage of the GMR sensor by position of the

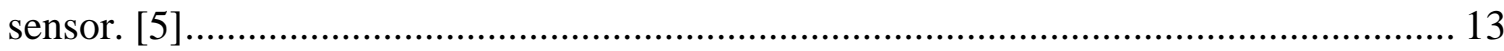

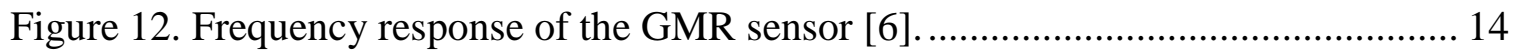

Figure 13. Power Module with integrated a GMR sensor [21] .................................... 15

Figure 14. Conventional way of using the GMR sensor for current-sensing. (a) On the PCB. (b) Integrated into the power module......................................................... 16

Figure 15. Illustration of the planar module. (a) Lay-up of the planar module. (b)

Packaged planar module.

Figure 16. Electrical resistance vs. Applied field.

Figure 17. Lay-up of the thin film layers of GMR material (a) normal condition (b) with external magnetic-field

Figure 18. Structure of GMR sensor (a) GMR resistor in the Serpentine structure (b)

Magnetic-field sensor in the form of Wheatstone bridge

Figure 19. Test board of the GMR sensor on a same dimension substrate as real module

Figure 20. Experiment setup for temperature response of the GMR sensor.................... 23

Figure 21. Measurement result of the Vout vs. Temp with different input voltage ......... 23

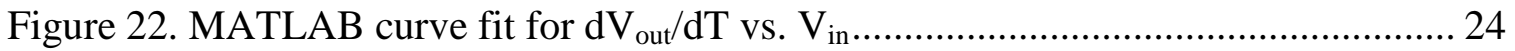

Figure 23. Experiment setup for evaluation of base resistance vs. temperature.............. 25

Figure 24. Experiment result of the base resistance vs. temperature.............................. 26

Figure 25. Block diagram of the temperature error compensation. ............................. 27

Figure 26. Compensated output voltage of the GMR sensor........................................ 27

Figure 27. The phase leg of the voltage source inverter ............................................. 30 
Figure 28. Packaging process of the planar power module

Figure 29. FEM Simulation model for flux density analysis

Figure 30. Magnetic-flux distribution on the copper trace with frequency sweep from 1

$\mathrm{Hz}$ to $100 \mathrm{kHz}$

Figure 31. Transient simulation model. (a) Simulation model. (b) Current excitation to the copper bars.

Figure 32. FFT of two current excitations (a). FFT of current 1. (b) FFT of current 2... 35

Figure 33. Observed magnetic-flux density in the middle of current paths. (a)

Magnetic-flux density vs. time. (b) FFT analysis of the magnetic-flux density.... 36

Figure 34. Simulation result with various frequency conditions. (a) Flux distribution on the observation plane $X$. (b) Magnetic-flux density versus frequency........................... 37

Figure 35. Current conduction paths of planar power module ..................................... 38

Figure 36. Magnetic-flux density observation at biased locations. ................................ 39

Figure 37. Magnetic-flux density at two biased points; (a) Point 2. (b) Point 3.............. 39

Figure 38. Double pulse tester setup ................................................................... 43

Figure 39. Waveform illustration of the double pulse tester. From Top: Inductor current, collector-emitter voltage, and gate voltage. 44

Figure 40. Switching sequence of the double pulse tester. (a) Low IGBT conducts and inductor current builds up. (b) IGBT turns off and inductor current freewheels through

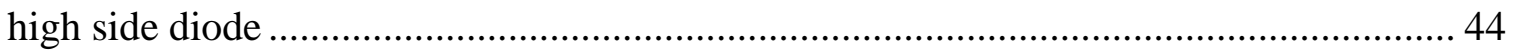

Figure 41. The preliminary GMR sensor test board. ................................................ 45

Figure 42. The output voltage waveform of the GMR sensor. (a) Measured signal. (b)

Zoom in the switching transient................................................................................ 46

Figure 43. Schematic of the instrumentation amplifier. .............................................. 47

Figure 44. Frequency response of the INA333 instrumentation amplifier. (a) Gain curve.

(b) CMRR curve.

Figure 45. Fabricated signal-conditioning circuit. (a) Schematic of the

signal-conditioning circuit. (b) Fabricated board on a PCB.

Figure 46. Output of the signal-conditioning circuit. (a) Comparison with the current

probe. (b) Zoomed in picture of transient moment. .................................................. 50

Figure 47. The waveform of the preliminary over-current protection circuit.................. 51

Figure 48. Schematic of the signal-conditioning circuit........................................... 52

Figure 49. The complete gate driver board with over-current protection. (a) Test board. (b)

Measured waveform with over-current situation.................................................... 54 


\section{LIST OF TABLES}

Table I. Summary of the current-sensing methods review ............................................... 11

Table II. Dimensions of the Planar Power Module....................................................... 31

Table III. Component list of the Double pulse tester...................................................... 42

Table IV. List of components of the gate driver circuit.................................................. 52 


\section{Chapter 1. INTRODUCTION}

\subsection{Motivation and Objective}

Conventional wire-bonded power modules showed limitation for high power applications mainly because of their poor thermal-management capability [1],[2]. Planar power modules (Figure 1) are a promising packaging alternative for high power applications thanks to their lower parasitic inductance, lower thermal/electrical resistance, and double-sided cooling capability. Integration of over-current protection circuit into a planar power module has been a challenge owing to the limited space available [11],[22].

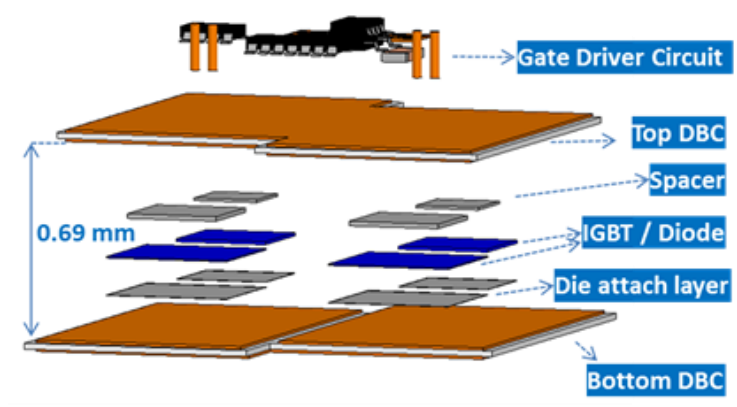

(a)

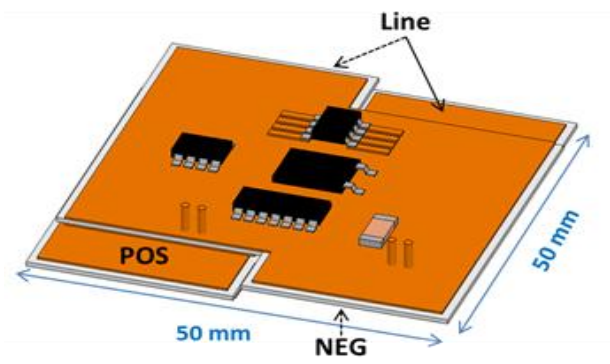

(b)

Figure 1. (a) Lay-up of a planar module; (b) planar power module after assembly.

Thus, a proper current-sensing method suitable for planar power module had to be identified. Several current-sensing methods were reviewed based on the following parameters:

- High bandwidth

- High reliability

- Compact size

- Consistent measurement over wide range of temperature

- Repeatable packaging process

- Low extra cost for integration 
The bandwidth of the sensor should be high enough to sense at the switching frequency of the current. The sensor should be durable considering the application of the planar module is the automobile. The coolant temperature of the automobile is usually $105^{\circ} \mathrm{C}$, and the ambient temperature seen by the current sensor is even higher by the influence of the additional power loss from switching devices [6]. The consistent measurement capability over wide range of operating temperature is important to integrate the current sensor into the module. Above all, the compact size of the sensor is important to truly integrate a current sensor into the module. Some current-sensing methods are reviewed based on the above parameters, and categorized according to their characteristics in Section 1.2 [17],[18]. The GMR sensor (Figure 2) was chosen as an integratable current-sensing method.

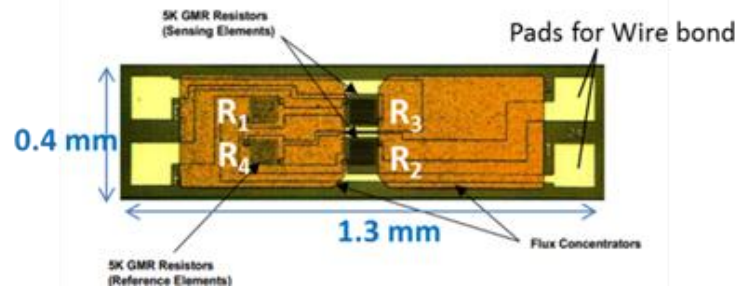

(a)

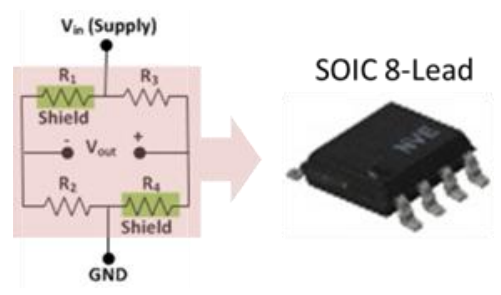

(b)

Figure 2. (a) GMR sensing element in die form; (b) schematic of the GMR sensor in a standard IC package [32].

As mentioned earlier, the GMR sensor in the planar module is highly influenced by the change of junction temperature. The measurement error that came from the varying ambient temperature was experimentally measured, and the measurement result is shown and analyzed in Section 2.2. The GMR sensor is a magnetic-field detector, so the magnetic-flux distribution of the planar power module had to be very carefully analyzed [5]. Chapter 3 shows the magnetic-flux distribution based on the finite-element analysis (FEA) using Maxwell 15, as well as the optimum location of the GMR sensor in the 
planar power module. Based on the simulation result, a test board was fabricated as designed, including the GMR sensor on top. Preliminary measurement result of the GMR sensor contained excessive noise that had to be attenuated to be used for over-current protection. The design of the signal-conditioning circuit and over-current protection circuit is reported in Chapter 4. The last section summarizes the paper and outlines future improvements.

\subsection{Review of Current-sensing Methods}

There are many kinds of current-sensing methods that can be used for power modules. The current-sensing methods can be divided into two main categories by measurement topology. The simplest and popular method is measuring Ohmic voltage drop by connecting a known resistor in series with a current carrying conductor. Another popular method can be categorized as magnetic-field sensing method. The magnetic-field is a result of current flow, so by measuring magnetic-field around of the current path the current information can be found. This method is especially popular when isolation is required, such as high power applications. This chapter will review several current-sensing methods by measurement topologies, and cite pros and cons of each sensing method. Finally, the best current-sensing method for the planar power module was determined.

\subsubsection{Shunt Resistor}

The Shunt resistor method is a very popular method for its simple operating principle and ease of use. Figure 3(a) illustrates the resistor in series with the current carrying conductor. Since the resistance of the Shunt resistor is very accurately known, the current flows in resistor can be calculated by measuring the voltage drop on the resistor. Since the ideal Shunt resistor is pure resistor, the bandwidth is up to several $\mathrm{MHz}$ which is very 
wide bandwidth compared with other current-sensing methods. The Shunt resistor has some error factors which come from the limitations of the material properties and geometry of a sensor. All resistive materials have temperature coefficient of resistance (TCR), and it will result errors over wide range of operating temperature. Also the geometry of the Shunt resistor can induce stray inductance, and it will induce the additional inductive voltage drop which will influence the output voltage measurement. Another important disadvantage of the Shunt resistor method is additional power loss and lack of galvanic isolation. The current flowing through the resistor always results $I^{2} R$ loss, and the loss gets bigger as sensing current goes high. Also the resistor should be connected in series with the current carrying conductor, so there is no isolation from the power stage to the sensing circuit. Compared with other magnetic-field sensing methods which will be reviewed in next paragraph, the compact size and DC to high bandwidth of the Shunt resistor is advantageous for the module integration, but it always comes with safety and additional power loss issues. Therefore, it should be carefully compared with other current-sensing methods to see which advantageous factors can trade off some disadvantages.

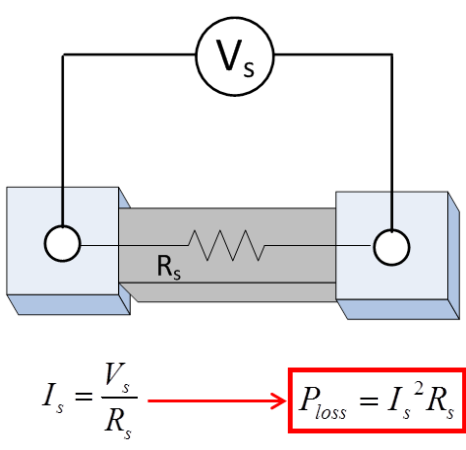

(a)

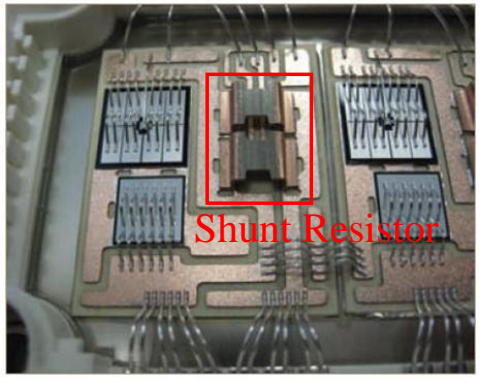

(b)

Figure 3. (a) The schematic of the Shunt resistor. (b) Shunt resistor in a commercial power module (Siemens SINAMICS G120). 


\subsubsection{Hall Effect}

The Hall element delivers an output voltage which is linear with the magnetic-flux density passing through the Hall element. When the magnetic flux is applied in a direction of perpendicular to the Hall element, the charges in the Hall element experience the Lorentz force, and these charges are concentrated at the surface of the Hall element. These charges establish the electric field, and the electrical potential between two surfaces has linear relationship with the applied magnetic-flux density that is related to the applied current in a conductor. Some Hall elements are chip-packaged, and they can be directly placed around current-carrying conductors to sense the magnetic-field density. However, this method does not provide strong enough magnetic-flux density to be sensed by Hall elements. Usually, Hall elements are placed in the air gap of a toroidal flux concentrator to increase the magnetic-flux density. Therefore, Hall-effect sensors usually have a toroidal core, and the current-carrying conductor has to go through the center of the core. The Hall-effect sensors have high accuracy, less than $2 \%$ measurement error, can sense dc current, and has moderate bandwidth, but the necessity of the core results in bulkiness which is unattractive for module integration. Even though the Hall-effect sensor is the most popular magnetic-field sensing method, it is not desirable for planar module integration.

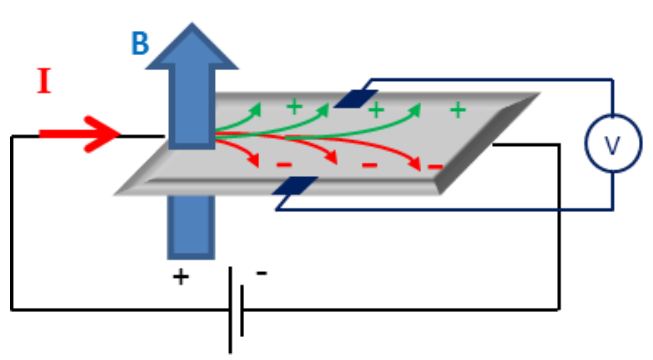

(a)

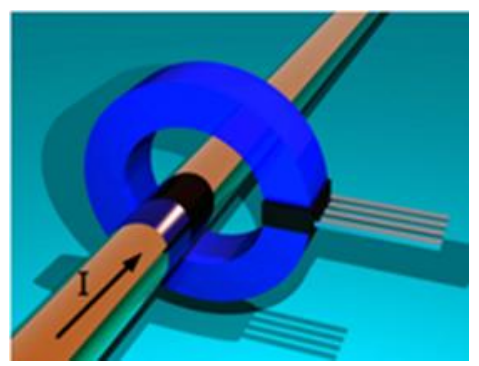

(b)

Figure 4. (a) The operating principle of the Hall Element. (b) The Hall Effect sensor with toroidal flux concentrator. 


\subsubsection{Current Transformer}

The current transformer is one type of transformer that has one turn on the primary side and several on the secondary side. The sensed current goes through the window of the magnetic core, whereas the secondary current goes through a resistive load that is a burden, for the voltage across the known resistance is reflected into the circuit for which the current is sensed. However, the current transformer has limitation for integration into the power module. The current transformer requires a magnetic core, and the need for a core limits the operating temperature of the sensor. Therefore, the current transformer is not a desirable current-sensing method for power-module integration.

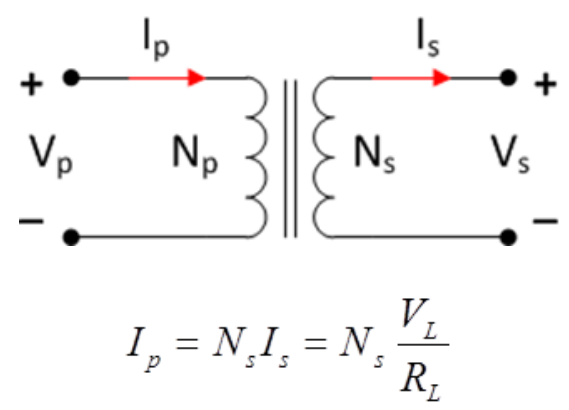

(a)

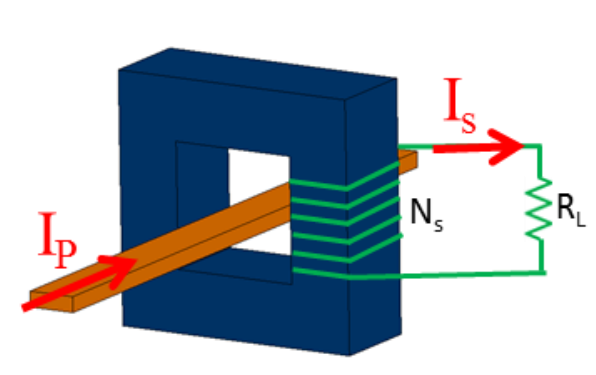

(b)

Figure 5. (a) Operating principles of the transformer. (b) Illustration of the current transformer in use.

\subsubsection{Anisotropic Magneto Resistive (AMR) Sensor}

The AMR sensor is one type of magneto resistive sensor that changes its resistance when it is exposed to the magnetic-field. The resistance of the AMR sensor changes by the angle between current and magnetization vector. The AMR sensor consists of Permalloy and the Permalloy is magnetized by external permanent magnets as shown in Figure 6. With an external power supply, the current $\mathrm{I}_{\mathrm{s}}$ induced which is in parallel with 
the magnetization vector $\overrightarrow{\mathrm{M}}$ (Figure 6(a)). In this case the angle between current and magnetization vector is zero, and resulting resistance is the maximum as the graph in Figure 7. When the external magnetic-field is applied, the magnetization vector is being biased as shown in Figure 6(b). In this case, the angle between current and biased magnetization vector is non-zero, and the resistance of the Permalloy reduces as graph in Figure 7.

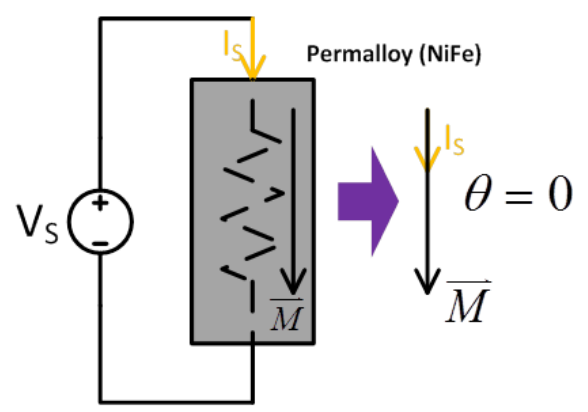

(a)

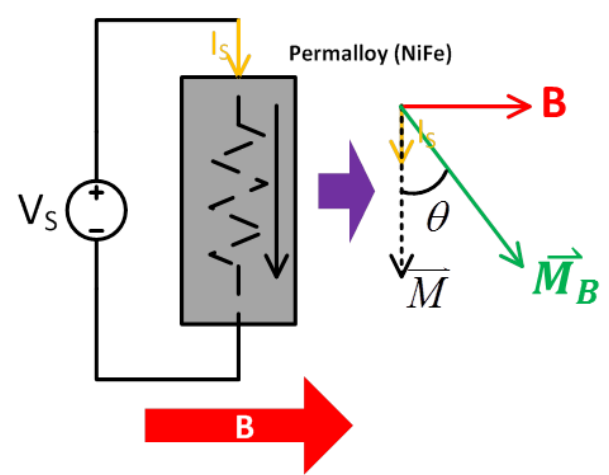

(b)

Figure 6. Operating principles of the AMR sensor. (a) Without external magnetic-flux. (b) With external magnetic-flux

Based on the described AMR effect, commercial current sensors were fabricated with special fabrication process. During the Permalloy fabrication process, the aluminum strips were layered with $45^{\circ}$ angle, which called as Barber Pole structure.

In this case, when the current is applied by power supply, the intrinsic characteristics of current that trying to find shortest path makes current to be biased with $45^{\circ}$ angle as illustrated in Figure 8(a). Four AMR resistors with Barber Poles were laid out in the Wheatstone bridge with different direction of the alumina strips which will result different bias angles. The yellow arrows in Figure 8(b) shows the intrinsic bias angle of each AMR resistor and the black dash arrow shows the intrinsic magnetization vector of 
the AMR resistor. The primary current to be sensed will be forced to flow beneath of four AMR resistors, following the U-shaped current trace in Figure 8(b).

If primary current starts flowing underneath of the AMR sensors, the magnetization vector will be biased as influence of magnetic-flux induced by the primary current. Finally, the angle between the supply current, and magnetization vector will be changed as shown in picture. In the example case in the picture, the R2 and R3 will have less resistance than remaining resistors, and the output voltage will be linearly changed by the resistance changes. The AMR sensor can measure from DC current and the bandwidth is up to $1 \mathrm{MHz}$, which is exceptionally higher than other magnetic-field sensing methods.

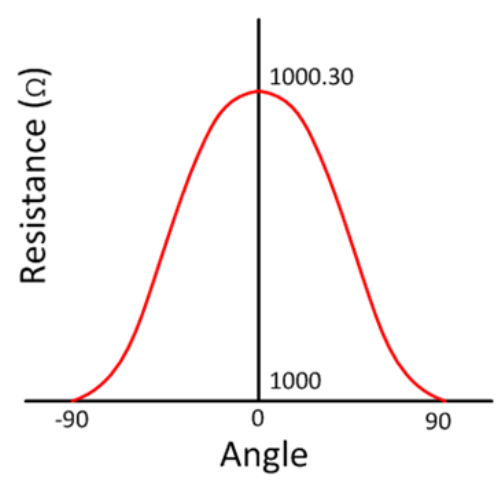
The primary current trace and AMR resistors have galvanic isolation, and the volume of the sensor is also only about $25 \%$ of Hall sensors or current transformers. Therefore, the AMR sensor can be a strong candidate for the power module integration. 

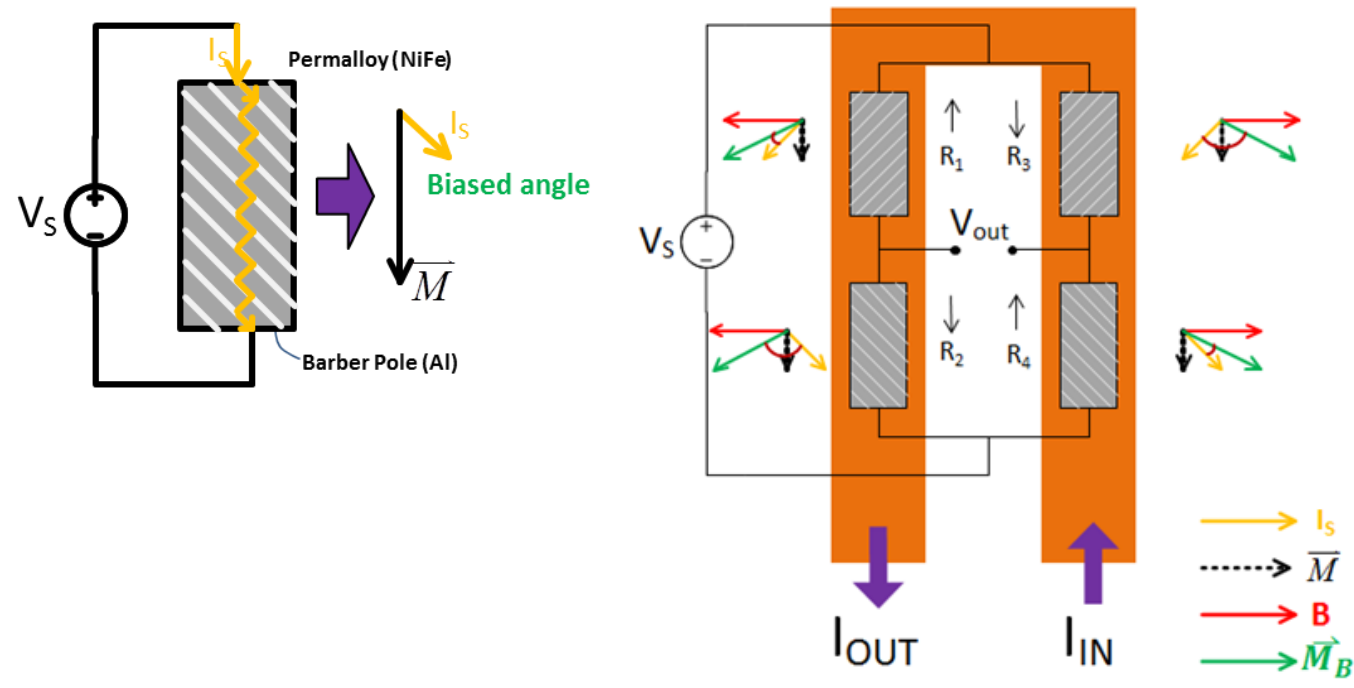

(a)

(b)

Figure 8. The structure of the AMR current sensor. (a) Fabrication of the AMR resistor with Barber Poles. (b) The AMR current sensor in the Wheatstone bridge structure.

\subsubsection{Giant Magneto Resistive (GMR) Sensor}

The GMR resistor is constructed from a type of material that changes its resistance when it is exposed to the magnetic field. The GMR resistor has a preferred axis of sensitivity, and when the magnetic field is applied in that direction, the GMR effect is maximized which is up to $16 \%$ of the base resistance. Figure 9 shows the relationship between resistance changes of GMR resistor and applied magnetic field.

Considering the resistance change of AMR sensor is up to $2 \%$, the GMR resistor can give wider sensing range with higher resolution than the AMR sensor. The bandwidth of the GMR is from dc to $1 \mathrm{MHz}$, and the operating temperature is from $-40^{\circ} \mathrm{C}$ to $150^{\circ} \mathrm{C}$. Both bandwidth and the operating

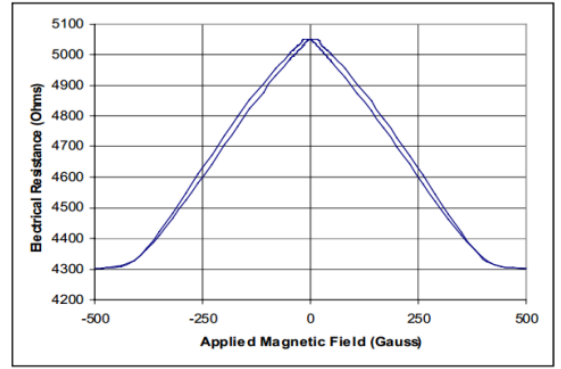

Figure 9. Relationship between GMR resistor and applied magnetic field. 
temperature are superior compared with conventional magnetic-field sensing methods. The compact size of the sensor is ideal for module integration. The packaged form has standard SOIC 8-lead package, and the die of the GMR sensing element is only $1.3 \mathrm{~mm} \mathrm{x}$ $0.4 \mathrm{~mm}$. By virtue of these advantages, the GMR sensor was determined as an integrated current-sensing method.

\subsubsection{Summary of the Current-sensing Methods}

Several current-sensing methods were reviewed to find the best option for the planar module integration. The important specifications for module integration were mainly reviewed in Table I. The targeting application has maximum current rating of $150 \mathrm{~A}$, so commercial current sensors that can measure up to 200 A were reviewed. Since the sensor measures the switching current, the bandwidth of the sensor is important parameter to see the high frequency transient components. If the current sensor is integrated into the power module, the junction temperature of the switching device can result a big temperature variation to the current sensor. The current sensor should be able to operate at high temperature, and the measurement error resulted by temperature variation should be small enough to minimize the error. Since the small footprint compared with conventional power module is one of the important advantages of the planar power module, the compact size of sensor is also important factor to keep the advantage of the planar module. Figure 10 shows the size comparison of commercial current sensors for each sensing topology. The current-sensing methods that require the magnetic core have relative bigger size than other current-sensing methods, and they also have low operating temperature by the material property change of the magnetic core. 
Table I. Summary of the current-sensing methods review

\begin{tabular}{|c|c|c|c|c|c|c|}
\hline & $\begin{array}{c}\text { Sensing } \\
\text { Range }\end{array}$ & $\begin{array}{c}\text { DC } \\
\text { Capability }\end{array}$ & Bandwidth & Accuracy & $\begin{array}{c}\text { Operating } \\
\text { Temperature }\left({ }^{\circ} \mathrm{C}\right)\end{array}$ & Isolation \\
\hline Shunt & Variable & Yes & $10 \mathrm{MHz}$ & $1 \%$ & -65 to 170 & N/A \\
\hline $\begin{array}{c}\text { Hall } \\
\text { Effect }\end{array}$ & $\begin{array}{c}\text { DC to } \\
200 \mathrm{~A}\end{array}$ & Yes & $150 \mathrm{kHz}$ & $0.5 \%$ & -40 to 80 & $2.5 \mathrm{kV}$ \\
\hline CT & $\begin{array}{c}\text { Up to } \\
200 \mathrm{~A}\end{array}$ & No & $\begin{array}{c}10 \text { to } 600 \\
\mathrm{~Hz}\end{array}$ & $<1 \%$ & -20 to 60 & $7.4 \mathrm{kV}$ \\
\hline AMR & $\begin{array}{c}\text { DC to } \\
300 \mathrm{~A}\end{array}$ & Yes & $\begin{array}{c}\mathrm{Up} \mathrm{to} 1 \\
\mathrm{MHz}\end{array}$ & $1.3 \%$ & -40 to 105 & \\
\hline GMR & $\begin{array}{c}1 \mathrm{mT} \text { to } \\
10 \mathrm{mT}\end{array}$ & Yes & $\begin{array}{c}\text { Up to } 1 \\
\mathrm{MHz}\end{array}$ & $2 \%$ & -40 to 150 & \\
\hline
\end{tabular}

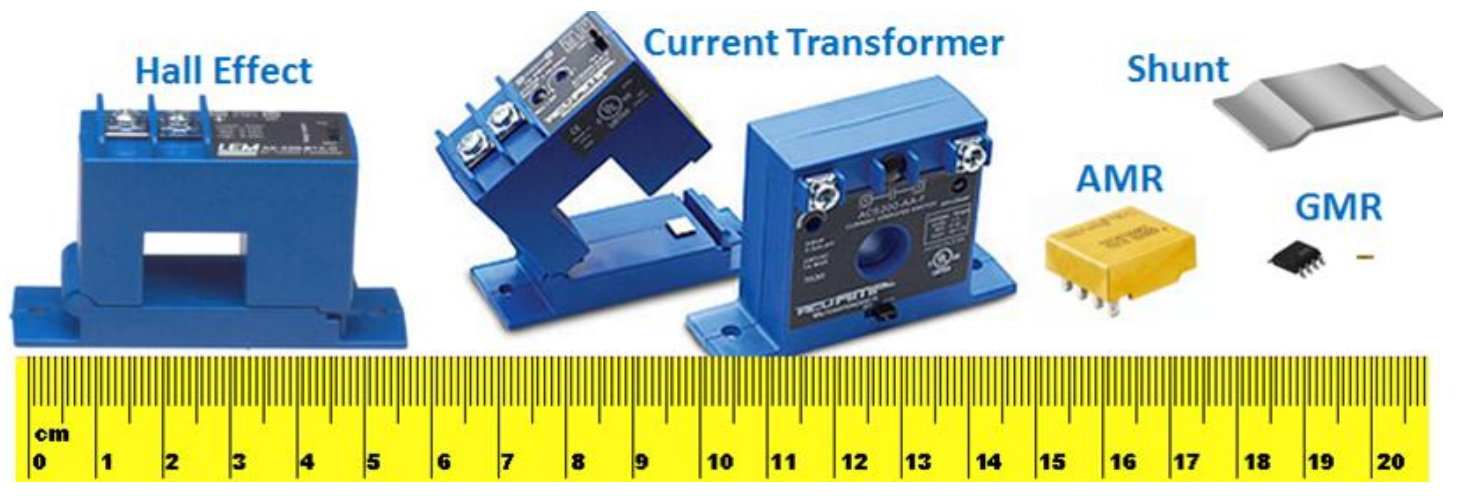

Figure 10. Size comparison of the commercial current sensors.

Since all current-sensing methods in Table I satisfy the current-sensing range, the bandwidth, operating temperature, and size were mainly considered to choose the current-sensing method. The planar module is under development for automotive application and the conventional switching frequency of the motor drive for automotive is about $5 \mathrm{kHz}$ to $10 \mathrm{kHz}$. So the current sensor is preferred to have higher bandwidth than this range, but the current transformer has too low bandwidth to satisfy the requirement. Next consideration for selection was the operating temperature range. The ambient temperature under hood of automotive is around $105^{\circ} \mathrm{C}$, and the actual temperature seen by sensor will be higher by the influence of the heat generated from the module. The Hall 
Effect sensor and AMR sensor are not able to satisfy this requirement so they also had to be excluded from the options. Rest of options were the Shunt resistor and the GMR sensor, but the Shunt resistor always results additional $I^{2} R$ power loss and does not have galvanic isolation that is preferred for high power applications. The GMR sensor has very high bandwidth, sensing current range can be adjusted by the position of the sensor, has high operating temperature compared with conventional current-sensing methods, and the compact size is one of the biggest advantage for the module integration. The GMR sensor was finally chosen as the current-sensing method, and detailed packaging process of the GMR sensor will be handled in this thesis.

\subsection{Literature Review}

The GMR sensor is a magnetic-field detector that can be used for many purposes, not just current-sensing. It can be used as position sensor, angle sensor, etc. This thesis is focusing on the GMR sensor as current sensor, so some literatures that used the GMR sensor as current sense were reviewed. This review will introduce the achievement from previous researchers, and show the obstacles when it comes to the planar module integration.

\subsubsection{Output voltage of the GMR sensor by sensing location}

The magnetic-flux density at a point is directly related to the distance from the center of a current carrying conductor. This paper showed possible method to increase the sensitivity of the sensor by a layout of the sensor on a PCB [5]. Figure 11(a) and (b) shows two different current conduction paths, one has current trace at the bottom layer of the PCB and GMR sensor at top layer, and the other case with detouring current trace on top of the GMR sensor. Since the magnetic-flux density is inversely proportional to the distance from the center of current carrying conductor, when current flows at the bottom 
layer of PCB, the output voltage is smaller than case (b). Figure 11(d) shows the measurement result of two different cases. The Figure 11(d) shows the influence of the sensor location on the wide current carrying trace with DC current. The DC current density will not be uniformly distributed in a wide trace, but will be concentrated at the middle of conductor. This is why when the sensor was placed in the middle of the conductor it showed higher output voltage compared with location along the edge. This paper showed how the sensitivity of the GMR sensor can be improved, but when it comes to the $\mathrm{AC}$ current measurement, the current distribution will be not uniform anymore. Also in this case, the targeting current was in a narrow current trace on a PCB. In this condition, the magnetic-flux density seen by the GMR sensor will be higher than the case of same amount of current flows in a wider trace.

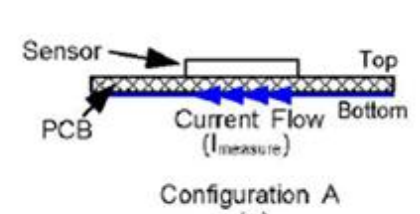

(a)

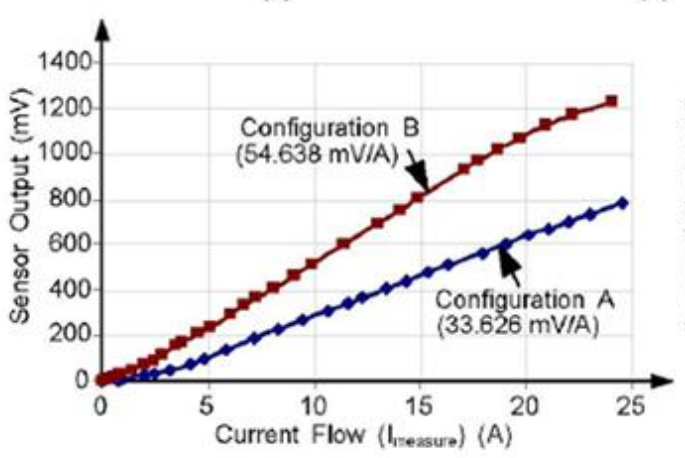

(c)

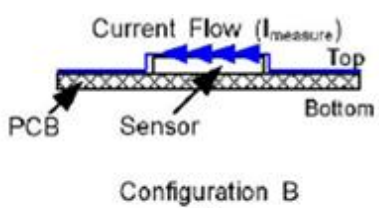

(b)

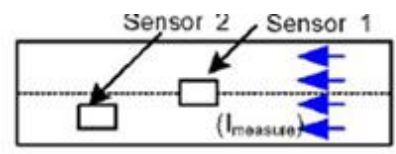

Configuration $\mathrm{C}$

(d)

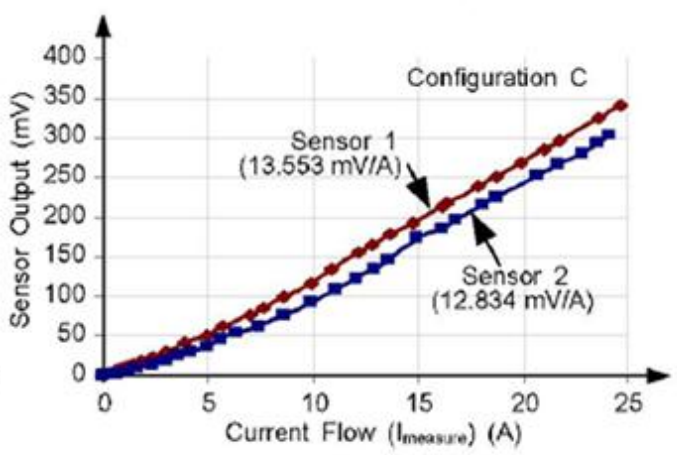

(e)

Figure 11. Literature review 1: The output voltage of the GMR sensor by position of the sensor. [5] 


\subsubsection{Influence of the Frequency to the GMR sensor}

The GMR sensor detects magnetic-field density, and the magnetic-field density of the $\mathrm{AC}$ current is directly related to the frequency of the current. The frequency response of the GMR sensor was measured by wrapping the GMR sensor with an air core coil to give various frequency magnetic-field excitations [6]. The Figure 12(a) shows the schematic of the experiment setup and the Figure 12(b) shows the measurement result. The vertical axis of top plot shows the normalized output voltage of the GMR sensor over the applied magnetic-field. The output voltage linearly increased with an applied magnetic-field, and the response of the GMR sensor dropped to $-1 \mathrm{~dB}$ at $400 \mathrm{kHz}$. Considering conventional Hall sensors have -1dB drop at $100 \mathrm{kHz}$, the GMR sensor has very wide bandwidth than competitive current-sensing methods.
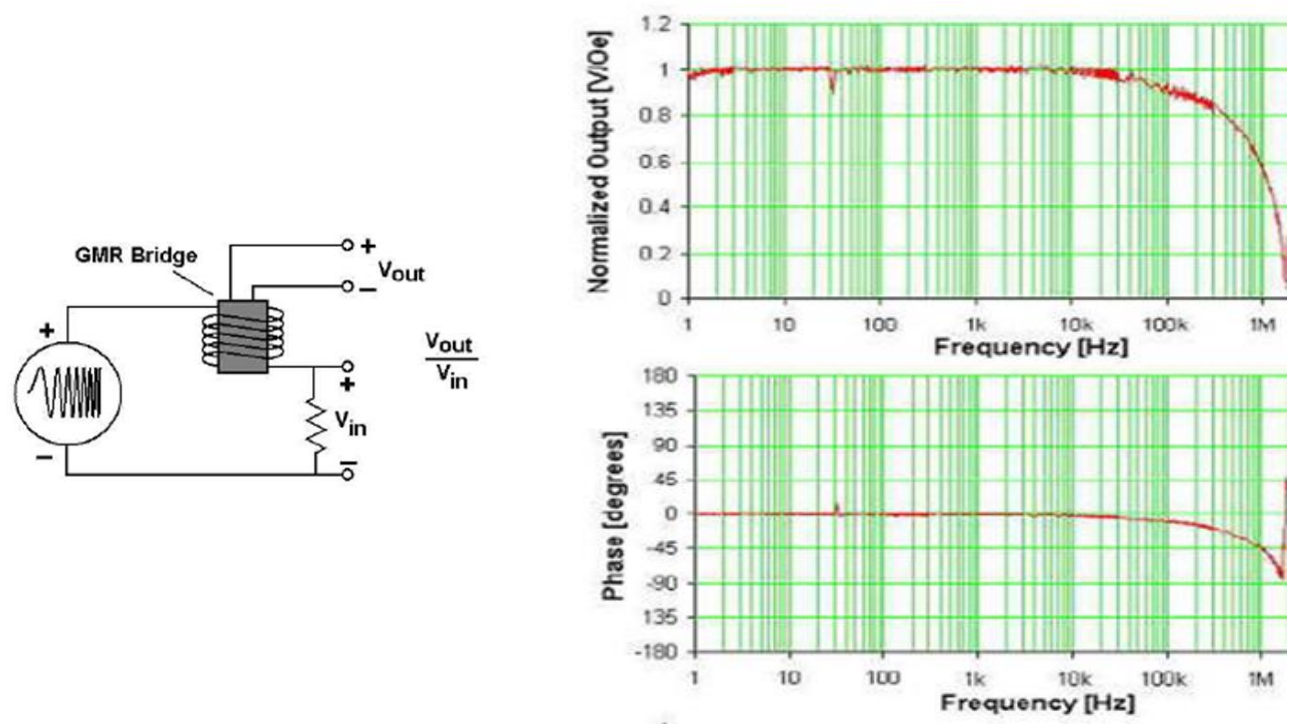

Figure 12. Frequency response of the GMR sensor [6].

\subsubsection{Power module integrated GMR sensor}

There was one paper that showing an integration of a GMR sensor into the power module. The module was $600 \mathrm{~V} / 20 \mathrm{~A}$ phase leg module, and it had gate driver circuit and 
a GMR sensor as current sensor. The module was packaged with Flex circuit that has flexible polyimide film between two copper layers at top and bottom. The Flex circuit had very thin copper layers and polyimide film which was several micrometer thicknesses. The bottom side copper layer of Flex circuit was used to make interconnection between power devices, and top side copper layer was used to place a current sensor and gate driver. Because current rating of the module was low, the current trace at the bottom side of Flex circuit had narrow width, and the thin polyimide film kept the distance from current trace to GMR sensor short. Therefore the magnetic-flux density could be well focused to the sensing element.

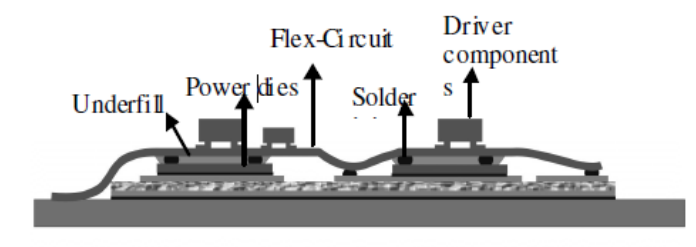

Econo3 base plate

b) Cross-sectional view of the flip-chip flex-circuit module
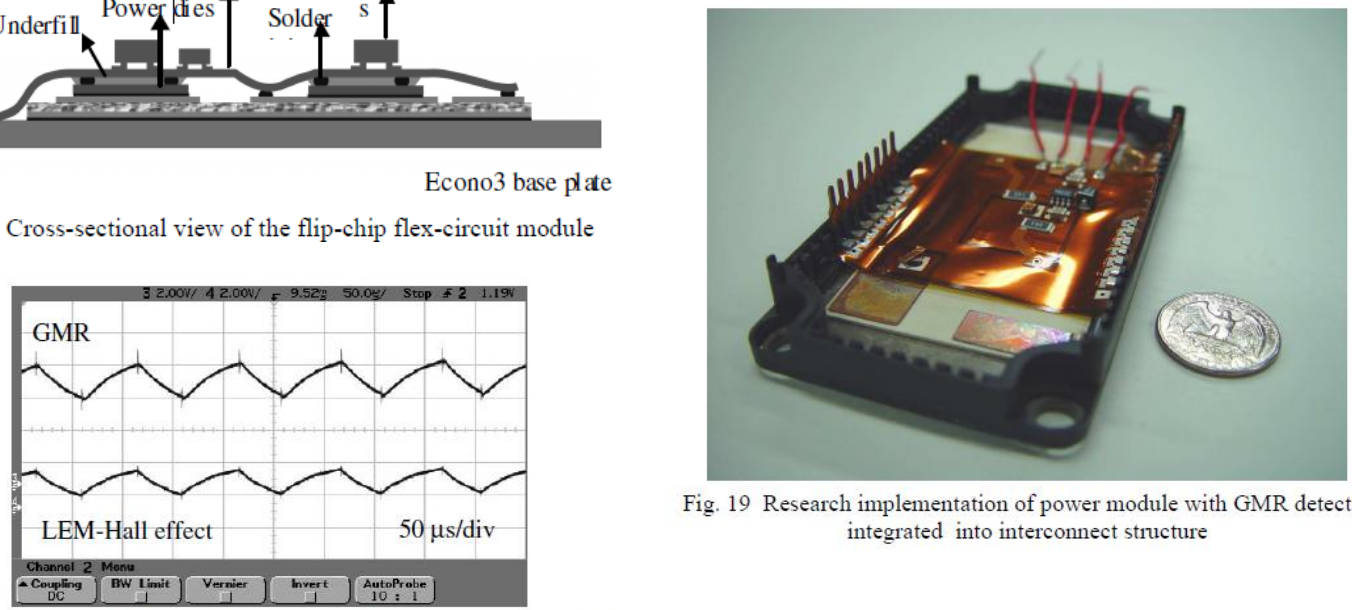

Fig. 19 Research implementation of power module with GMR detector integrated into interconnect structure

Fig. 15 Evaluation of integrated point field GMR detectors and classical LEM, closed loop Hall effect sensors [11]

Figure 13. Power Module with integrated a GMR sensor [21].

\subsection{Significance and Objective of This Study}

The GMR sensor is packaged in the SOIC 8 form, and it works best on a PCB level circuit packaging. Most of literature reviews showed integrating a sensor into the top 
layer of PCB with a power stage current trace at the bottom layer. This was possible because the primary current was low level, and the required current trace width was narrow enough that can be similar or smaller than the package of GMR sensor (Figure 14(a)). There was one research that integrated the GMR sensor into the power module. The Flex circuit that has thin polyimide film at the middle and thin copper layers at both top and bottom sides was used to make interconnection to the power dice, and also the current loop for a power stage. The GMR sensor was placed on the top surface of the Flex circuit so that the sensor can be placed directly on top of the current trace. In this way, the strong magnetic-field can be directly observed by GMR sensor.

From literature reviews, one can be noticed that all cases use GMR sensor for low current measurement, and the current carrying conductor is narrow and very close to the GMR sensor. Also both systems do not have nearby power devices or ICs that can generate excessive heat.

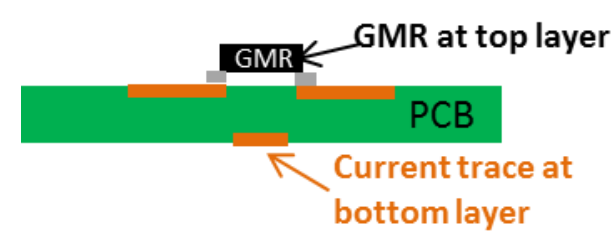

(a)

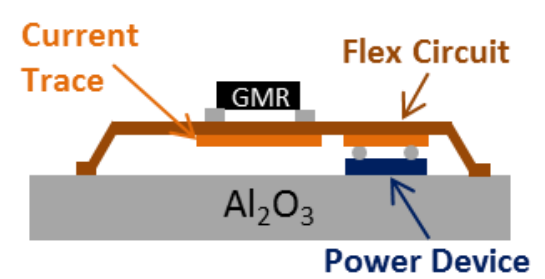

(b)

Figure 14. Conventional way of using the GMR sensor for current-sensing. (a) On the PCB. (b) Integrated into the power module.

However, in case of the planar power module, large size IGBTs were packaged that can handle up to $150 \mathrm{~A}$, and the copper pattern on the DBC substrate also got wider to be able to package large dice. When the AC current goes through the wide current trace, the current will be non-uniformly distributed in the conductor, because of the eddy-current effect. It will result non-uniformity of the magnetic-flux distribution, and it will require 
very careful positioning of the GMR sensor to get an accurate measurement result. The signal-to-noise ratio (SNR) for low current measurement is also a problem to be solved. If the current is high enough so that the magnetic-flux is well in a sensor's linear range, the signal ratio overwhelms the intrinsic measurement noise. However, if the current is low, the ratio of the measurement noise gets high, and the output voltage of the GMR sensor is hard to use without additional signal-conditioning circuit. Figure 15 shows the structure of the planar module. The top surface of the top side DBC substrate will have gate driver circuit, along with current sensor. Since the DBC has high thermal conductivity, the junction temperature of the power devices will influence the temperature of the GMR sensor as well. The measurement error of the GMR sensor over wide temperature range will be presented, and the solution for the error compensation will be presented in the thesis.

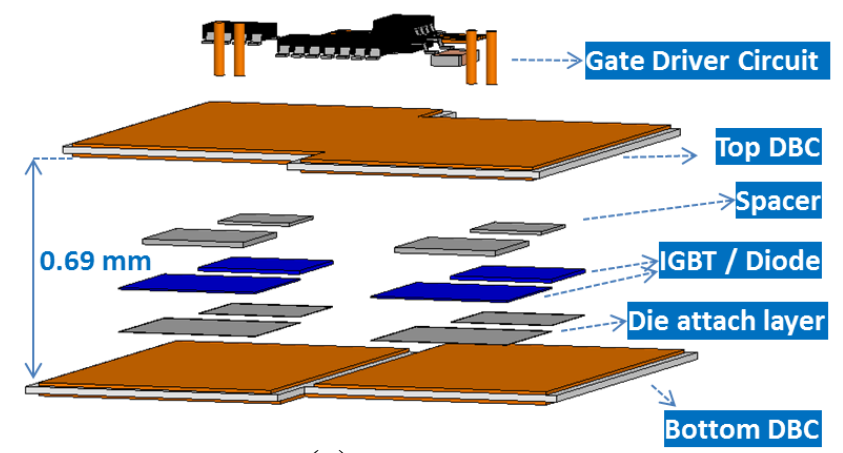

(a)

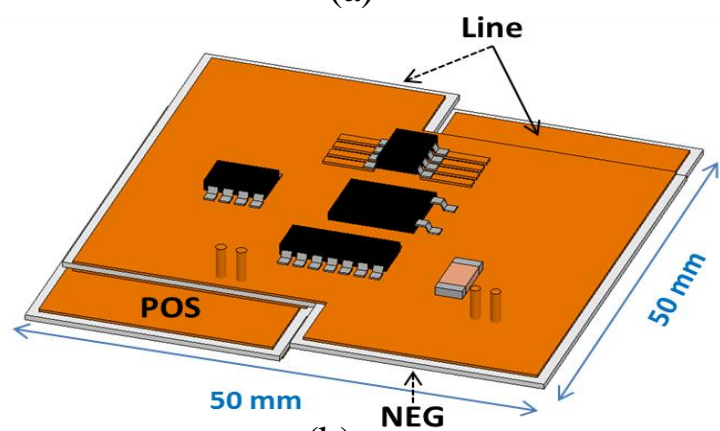

(b)

Figure 15. Illustration of the planar module. (a) Lay-up of the planar module. (b) Packaged planar module. 


\section{Chapter 2. Principles AND Characteristics OF GMR}

\section{SENSOR}

The GMR sensor is non-ideal for varying temperature. The high junction temperature of the power devices will influence the entire module's temperature, so quantifying the influence of temperature to the measurement accuracy was very important.

\subsection{Basic Operating Principles of the GMR Sensor}

For the applied magnetic-field to the GMR material, a resulting resistance change has omnipolar characteristics. Figure 16shows the relationship between the electrical resistance changes versus applied magnetic-field. Assuming there is no hysteresis, electrical resistance changes in a same way regardless the direction of the applied magnetic-field. This characteristic makes it easy when the GMR sensor is used for the over-current protection purpose. The details about this will be explained more in Chapter

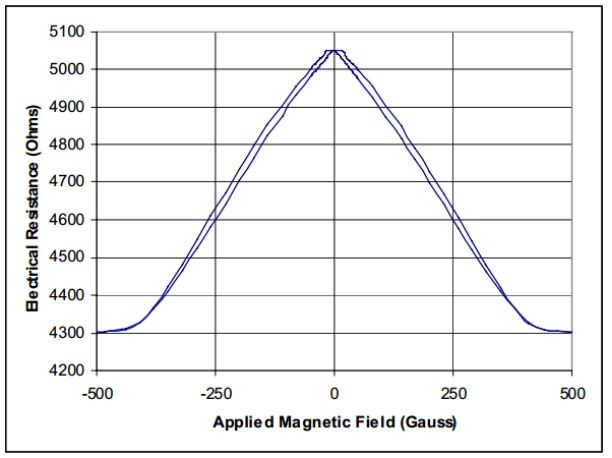
4.Another advantageous characteristic is the sensing capability of a constant field. This is

Figure 16. Electrical resistance vs. Applied field. the main advantage of GMR sensor compared with other inductive field sensors. Inductive magnetic sensors can sense only continuous change of the magnetic-field which means DC current cannot be measured. However, GMR sensor can measure constant magnetic-field thus GMR sensor can measure DC current as well.

\subsubsection{The Physics of the GMR Resistor}

The commercial GMR material is film layered material which consists of various alloys, such as nickel, iron, cobalt, and copper. The GMR material relies on anti-ferromagnetic 
coupling between the layers. Figure 17 shows the structure of GMR material. The conductive non-magnetic layer is sandwiched between magnetic material layers, and magnetic material layers at top and bottom are opposite each other. This structure enables the anti-ferromagnetic coupling between layers which enables maximized GMR phenomena. For the normal condition of the GMR material, the electrons in the magnetic material tend to scatter off at the interface of layers which results shorter mean free path length of the electron. However, when strong external magnetic-field is applied near GMR material, it will overcome the anti-ferromagnetic coupling. In this case, both top and bottom magnetic layers will have same spin, and electrons can move much easily cross the interface of layers. Comparing these two cases, when external magnetic-field is applied, mean free length of electron can be longer which will result a lower resistance of the GMR material. The anti-ferromagnetic structure helps to keep its magnetic-field sensing capability even if it is exposed to the excessive magnetic-field. Even if above linear region magnetic-field is applied to the GMR material, it goes back to normal operation when the large magnetic-field is removed. In this way, the GMR material can keep its characteristics for extremely large magnetic-field.

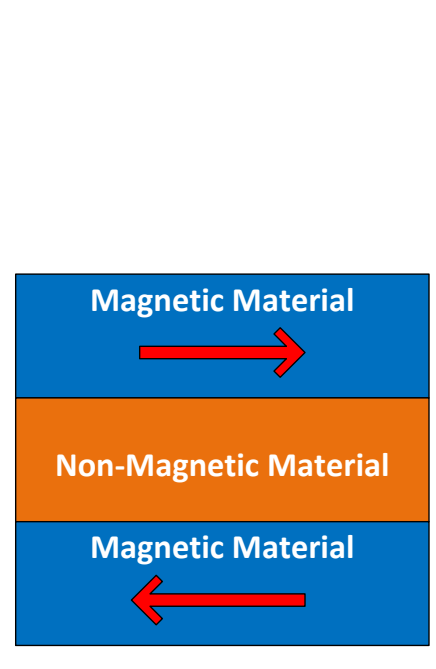

(a)

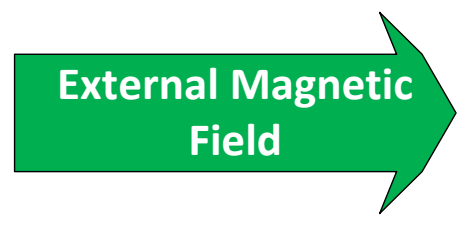

Magnetic Material

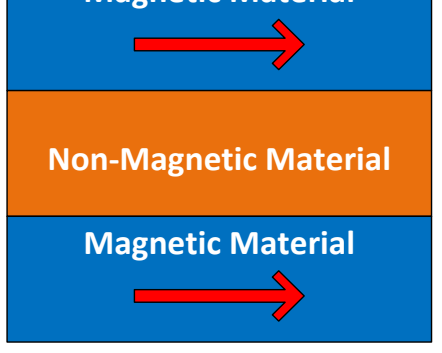

(b)

Figure 17. Lay-up of the thin film layers of GMR material (a) normal condition (b) with external 
magnetic-field

\subsubsection{Configuration as Current Sensor}

To maximize a resistance change to an applied magnetic-field, the practical GMR sensor was fabricated in the serpentine structure as shown in Figure 18(a). When a current flows in a perpendicular direction of the GMR resistor, applied magnetic-field goes through each layer of GMR resistor so that resistance change from one end to the other end can be maximized. The practical current sensor has the Wheatstone bridge structure as shown in Figure 18(b). Four GMR resistors form the Wheatstone bridge structure, and two GMR resistors located diagonally are magnetically shielded so that external magnetic-field will not change the resistance of these two shielded resistors. On the contrary, other two GMR resistors are located between flux concentrator so that the resistance change by applied magnetic-field can be maximized. Therefore, when supply voltage was applied between $V_{\text {in }}$ and ground, the output voltage $V_{\text {out }}$ will represent the magnetic-field information which refers to the current information. The output voltage of the Wheatstone bridge can be calculated as,

$$
V_{\text {out }}=\left(\frac{R_{4}}{R_{3}+R_{4}}-\frac{R_{2}}{R_{1}+R_{2}}\right) V_{\text {in }}
$$

The R3 and R4 will change their resistance when they are exposed to the magnetic-field.

Therefore, the change of this resistance should be studied for different inputs such as frequency response, temperature response, and sensing geometry. 


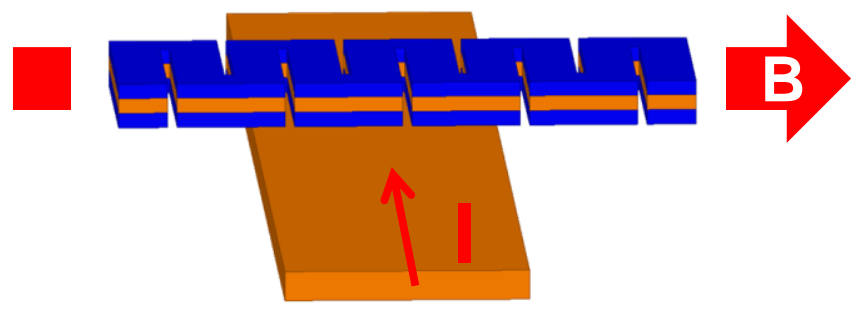

(a)

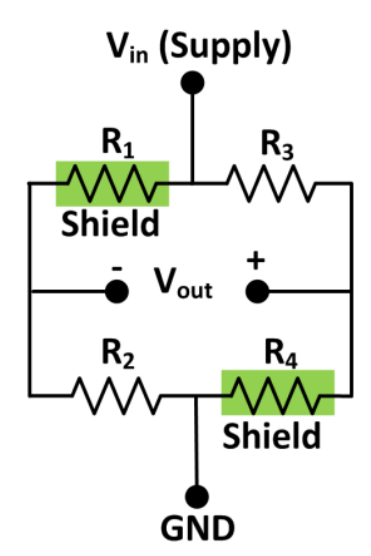

(b)

Figure 18. Structure of GMR sensor (a) GMR resistor in the Serpentine structure (b) Magnetic-field sensor in the form of Wheatstone bridge

\subsection{Thermal Characteristics}

The Wheatstone bridge structure of the sensor helps to keep the consistent measurement capability of the sensor for wide range of operating temperature [7]. Each GMR resistor has its own temperature coefficient of resistance (TCR), but four GMR resistors in the GMR sensor change their resistance in a similar rate, regardless it has shield or not. Therefore, when a constant voltage source is applied to the GMR sensor the current flow may change, but the output voltage will remain same in an ideal case [8]. The resistance that just mentioned is the base resistance of the GMR sensor, but there is another factor that influences the output voltage of the sensor by the ambient temperature. The GMR effect also influenced by temperature and literatures says that the rate of change per temperature is even higher than the base resistance of the GMR sensor. The temperature response of the GMR sensor will be tested by experiment, and the proper measurement error compensation method will be presented.

$$
\begin{aligned}
R_{\text {total }} & =R_{\text {base }}+R_{\text {GMR }} \\
\% G M R & =\mathrm{Ch} \text { ang e in resistance / M ica }
\end{aligned}
$$


The test board was fabricated to see the temperature response of the GMR sensor. Figure 19 shows the fabricated test board that can mound the GMR sensor on the top surface of DBC substrate with land pattern. The dimension of the DBC substrate was kept same as top substrate of the real module so that the test can imitate the actual operating condition of the module. The bottom side copper layer was also patterned as actual module that carries current in it.

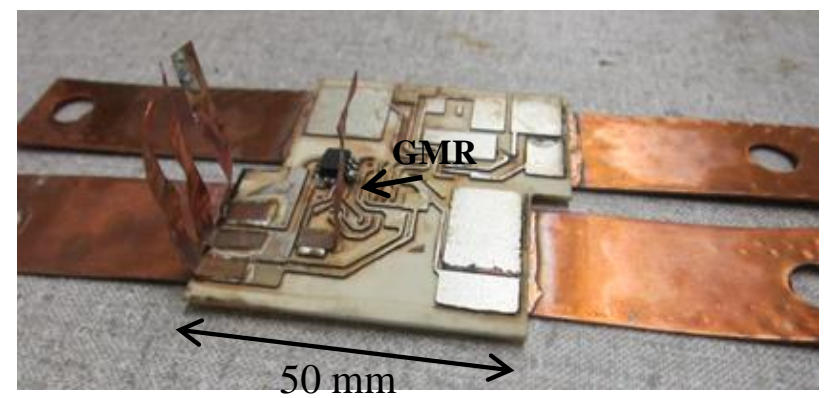

Figure 19. Test board of the GMR sensor on a same dimension substrate as real module

Figure 20 shows the experiment setup to see the temperature response of the GMR sensor. The test board was placed on the hot plate with a temperature controller, and a DC power supply was connected to the Vin+ and GND of the GMR sensor. The test was performed with constant $80 \mathrm{ADC}$ current, and the variable of the experiment was the supply voltage to the GMR sensor. The output voltage of GMR sensor and the temperature of the sensor were monitored by Agilent data acquisition equipment that connected to the computer. The thermocouple was attached to the lead of the GMR sensor, because the lead is the closest accessible point where close to the sensing element. After setting up the test bench, the temperature of the thermocouple and the supply voltage were incremented step by step, and the output voltage was monitored accordingly. 


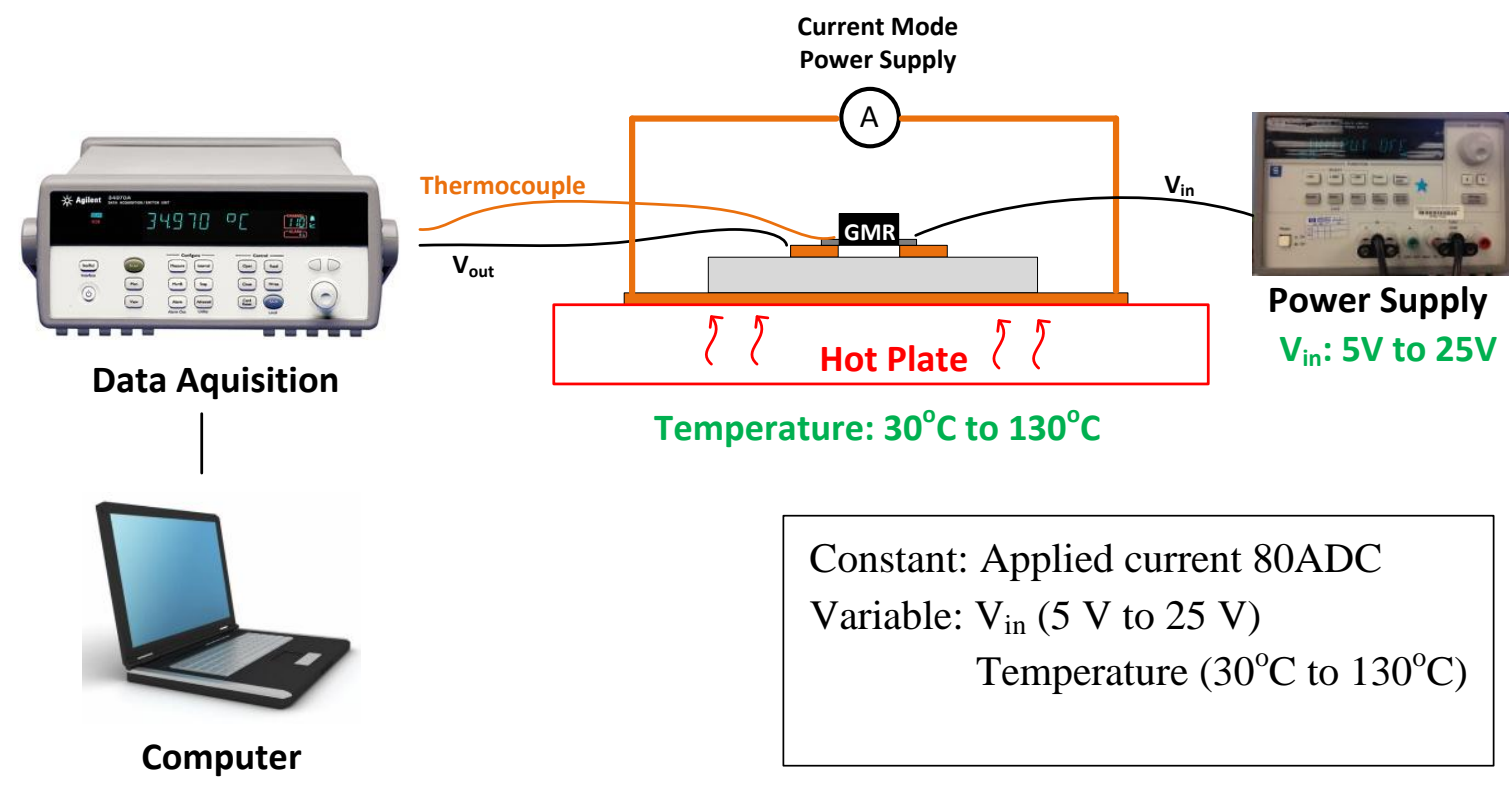

Figure 20. Experiment setup for temperature response of the GMR sensor

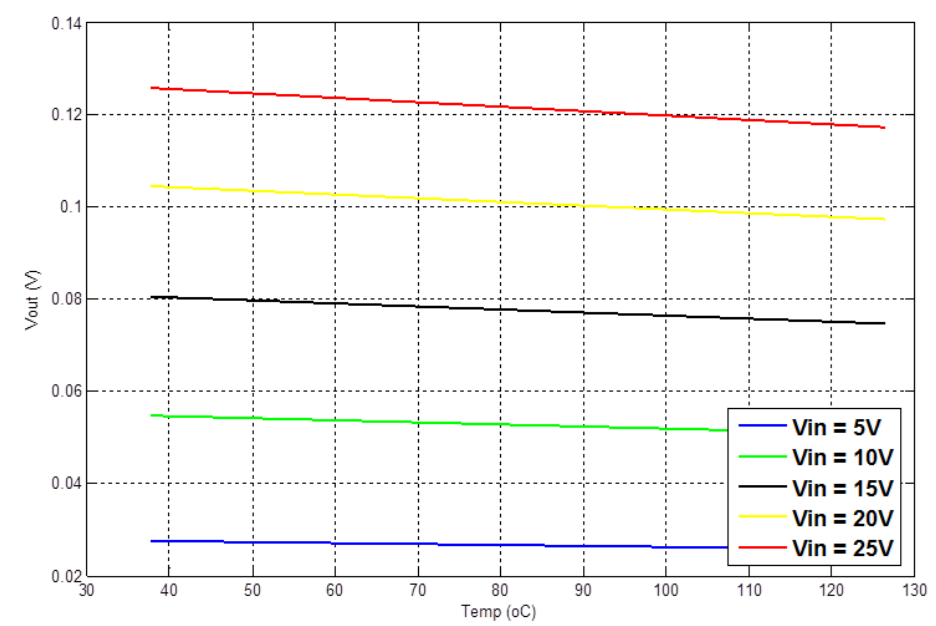

Figure 21. Measurement result of the Vout vs. Temp with different input voltage

Figure 21 shows the measured data from experiment setup. The output voltage versus temperature curves were plotted by different input voltages. The slope of each curve represents the linear relationship between temperature and output voltage of the sensor, 
and in an ideal case, the slope of each curve should be flat. However, all measurement showed negative slope that means the output voltage tends to be decreased as temperature increased, and also the slope of each curve showed variation by different input voltages. The linear equation that shows the influence of the input voltage to the rate of output voltage change per temperature was found by MATLAB curve fitting tool (Figure 22).

$\frac{\Delta V}{\Delta T}=\left[0.05819 V_{i n}{ }^{2}-5.306 V_{i n}+1.942\right] \cdot 10^{-6}$

The output voltage change over temperature change could be expressed as a function of input voltage. It means that the error of the output voltage can be calculated, if the temperature change of the GMR sensor is known.

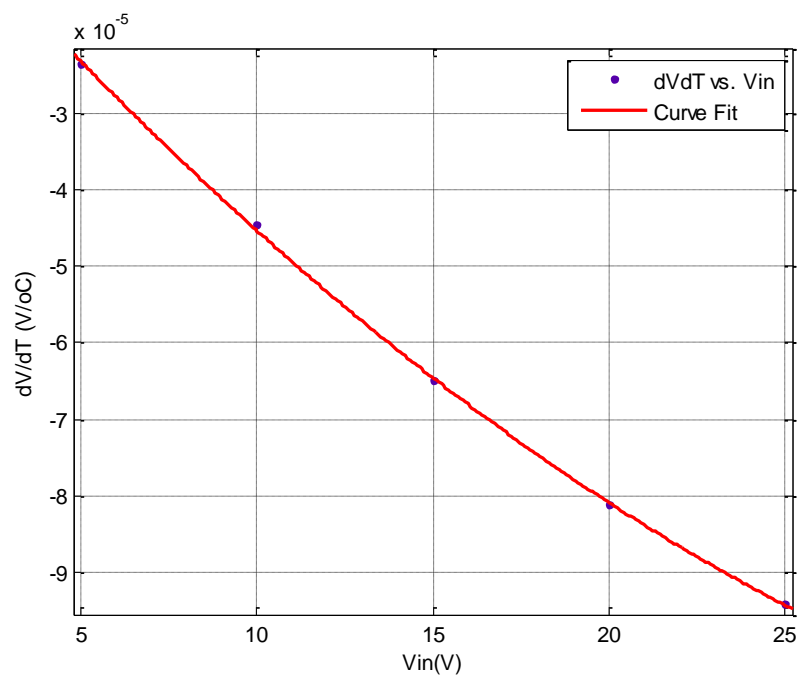

Figure 22. MATLAB curve fit for $\mathrm{dV}_{\text {out }} / \mathrm{dT}$ vs. $\mathrm{V}_{\text {in }}$

To be able to compensate the bias voltage of the output voltage, the temperature of GMR sensor somehow needs to be measured. This can be done by adding additional temperature sensor, such as thermal diode, but it will result an additional component in a module. The measurement result in Figure 21 shows the temperature response with the existence of the constant magnetic-field, and this term is including the change of both 
$\mathrm{R}_{\text {base }}$ and $\mathrm{R}_{\mathrm{GMR}}$. There are four GMR resistors in the GMR sensor, and two of them are magnetically shielded so these resistors will change its resistance only for the temperature variation. If resistance of the shielded resistor linearly changes its resistance over temperature, the TCR of the GMR resistor can be used for temperature sensing. To evaluate the temperature response of base resistance, the experiment was setup as shown in Figure 23. The GMR sensor was placed on the hot plate and the current mode power supply was connected to the $\mathrm{V}_{\text {in }}$ with $10 \mathrm{~V} / 1 \mathrm{~mA}$. In this way, the current flowing into the shield resistor is constant, and the resistance change can be calculated by measuring the voltage drop across the shield resistor.

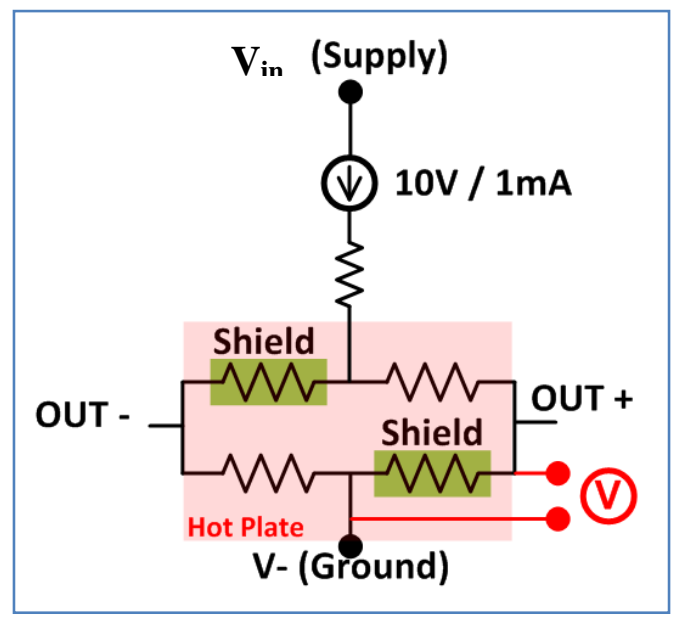

Figure 23. Experiment setup for evaluation of base resistance vs. temperature.

Figure 24 shows the experiment result. The voltage across shield resistor showed linear relationship with temperature change, and the linear equation could be found as,

$V_{\text {shield }}=8.96 \cdot 10^{-6} T^{2}+0.0048 T+4.088$

Since the applied current kept constant as $1 \mathrm{~mA}$, the base resistance change as function of temperature also can be found as,

$R_{T C R}=8.96 \cdot 10^{-3} T^{2}+4.8 T+4088$ 
It can be seen that the voltage across shield resistor is increasing by temperature. Considering the output voltage of the GMR sensor was decreasing as increase of temperature, it can be concluded that the GMR effect gets higher influence by the temperature than the base resistance. From this experiment, by measuring the voltage across the shield resistor, the temperature of the GMR sensor can be calculated using the derived equation.

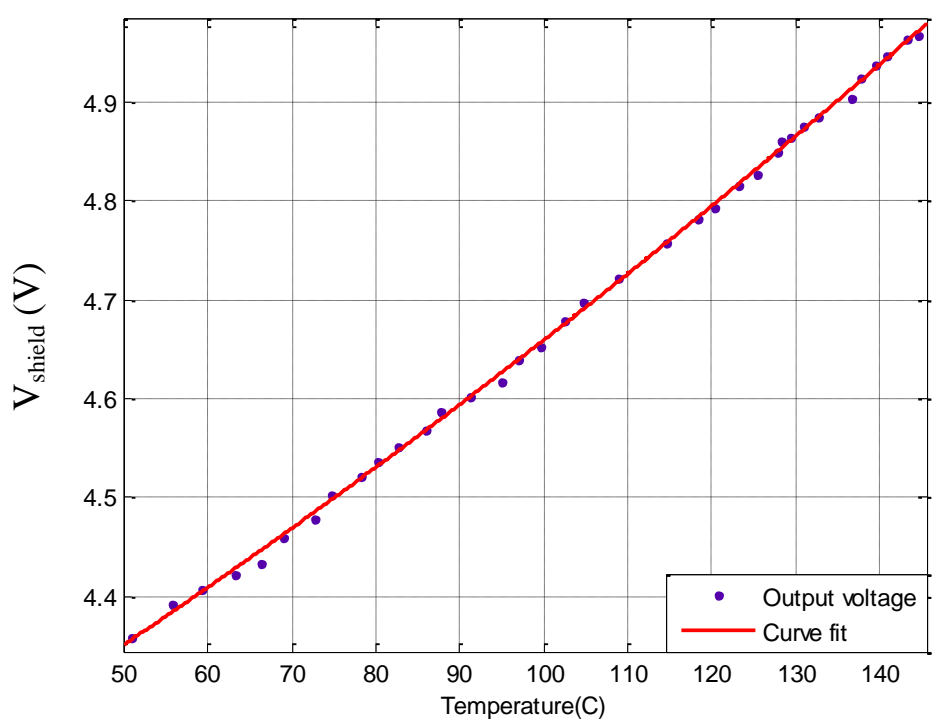

Figure 24. Experiment result of the base resistance vs. temperature.

By using the experiment results, the measurement error by temperature can be compensated. Figure 25 shows the block diagram of the temperature error compensation method. The voltage across the shield resistor will give the temperature information of the sensor, and the output voltage error by temperature can be calculated from the input voltage and sensed voltage at previous stage. The calculated output voltage error will be a negative number, so by subtracting the output voltage error to the measured output voltage, the compensated output voltage will be calculated. Figure 26 shows one example of the voltage compensation. When the temperature of the GMR sensor changed from 
$60^{\circ} \mathrm{C}$ to $120^{\circ} \mathrm{C}$, the output voltage of the sensor will be decreased. The calculated temperature change from the measured $\mathrm{V}_{\text {shield }}$ can estimate the error of the output voltage, and the estimated error voltage will compensate the output voltage of the GMR sensor. The red curve in Figure 26 is the compensated output voltage of the GMR sensor, and it is showing consistent output voltage from $60^{\circ} \mathrm{C}$ to $120^{\circ} \mathrm{C}$.

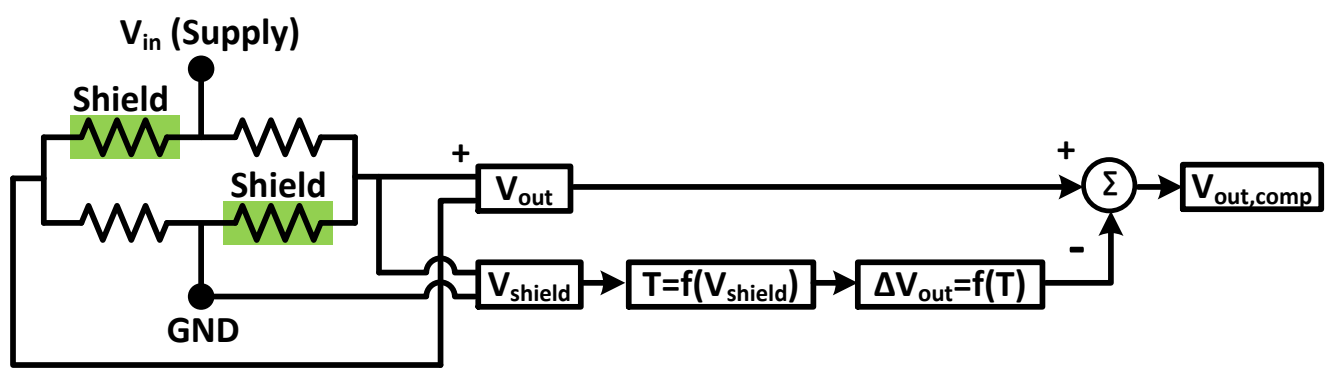

Figure 25. Block diagram of the temperature error compensation.

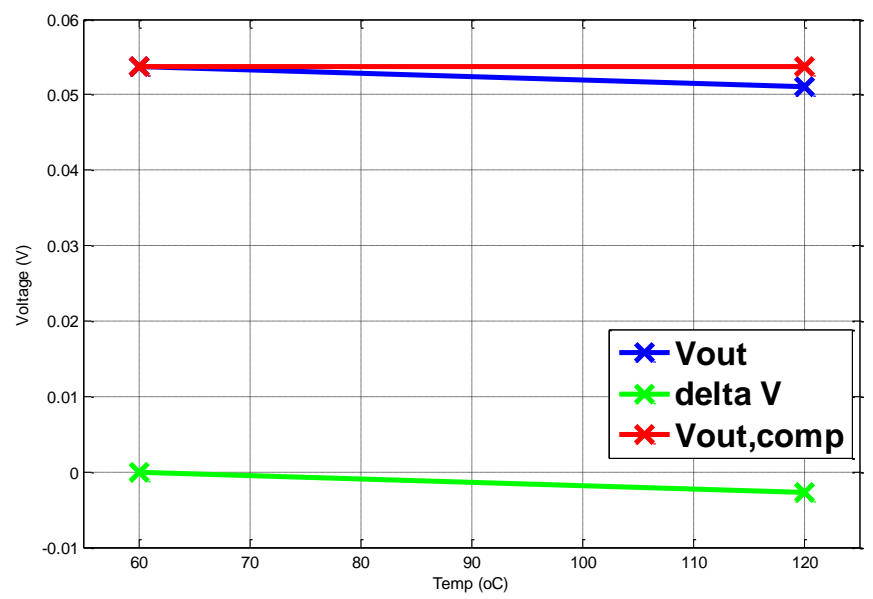

Figure 26. Compensated output voltage of the GMR sensor. 


\subsection{Conclusion}

The operating principle of the GMR sensor was reviewed and the temperature response of the sensor was experimentally measured and analyzed in this chapter. Because the GMR sensor will be packaged inside of the module, the power loss from switching devices will directly influence the operating temperature of the sensor. The experiment result showed that for $80 \mathrm{ADC}$ current measurements, the output voltage was changed by $5 \%$ for $60^{\circ} \mathrm{C}$ temperature change. For comparison with the conventional current-sensing methods, the shunt resistor will change its out voltage by $7 \%$ with same condition. The $5 \%$ error can be accepted or not by how much accuracy the system requires. If the percentage of error is not acceptable, the proposed real time temperature error compensation method can be used for higher accuracy measurement. 


\section{Chapter 3. Magnetic-flux Distribution of the Planar \\ POWER MODUle}

The GMR sensor detects the magnetic-flux around of it and gives that information in a voltage form. To predict an accurate measurement, the flux distribution of the planar module should be known for its actual current excitation. The current going through the trace will have a frequency, and the sensor should be able to detect all frequencies including a fundamental frequency and other harmonic frequencies. Also for the packaging point of view, using minimum number of devices is preferred to simplify packaging a packaging process and manufacturer cost. Since two current paths in the module conduct complimentary, only one GMR sensor was planned to be used by measuring both current information at the middle. To find the best location of the sensor, the magnetic-flux change at various points and frequencies were analyzed. This chapter will show the FEA simulation model of the module, and mainly discuss about the magnetic-flux distribution by different input factors such as frequency and location.

\subsection{Structure of the Planar Power Module}

As power demand to the power module increases, a conventional wire bond power module is reaching its limitation for high power operation. One side heat sinking is not enough to drain excessive heat generated by high current, and wire bond increases a loop inductance which will cause additional ringing. The proposed planar power module has many advantages compared with wire bond power module. The main difference is the front side wire bonds are replaced by a direct solder joint to a DBC substrate which is a symmetrical structure as back side. Since the front side of a chip is attached to the DBC substrate, both front and back sides are capable of heat sinking so that thermal management capability can be maximized. Additionally, a solder joint was replaced by 
the Nano-silver paste, which has four times higher thermal conductivity than the eutectic solder. These features can maximize the thermal management capability of the planar power module, and the maximum junction temperature can be significantly increased.

The targeting module to be packaged as planar structure was a phase leg of an inverter. Since the phase leg packaged power module can be used in many ways for different topologies, the phase leg of an inverter was planned to be packaged.

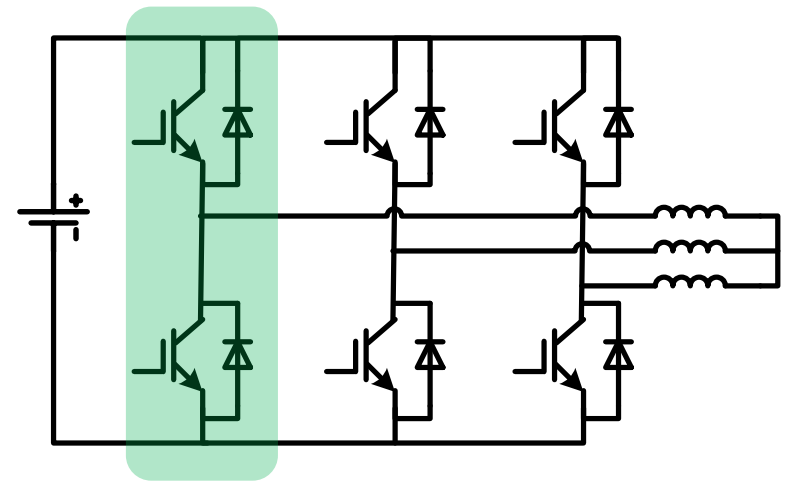

Figure 27. The phase leg of the voltage source inverter

Figure 28 illustrates the packaging process of the planar power module. The bottom DBC substrate was prepared by wet etching process, and Nano-silver paste was applied on the silver coated copper surface. Two IGBTs and didoes were placed on the silver paste, and another layer of Nano-silver paste was applied on the front surface of dice. Finally, the top DBC substrate placed on top, and attached by hot press process. Copper pins were used to make connections from gate and source pads to top copper, where gate driver will be placed. Top DBC substrate was laser drilled to make wire bond connection from gate/source pads to top copper. This was temporary solution for preliminary version of the module, but for the future modules gate/source pads will also be connected to the substrate by Nano-Silver paste so that the reliability of the module can be maximized. 


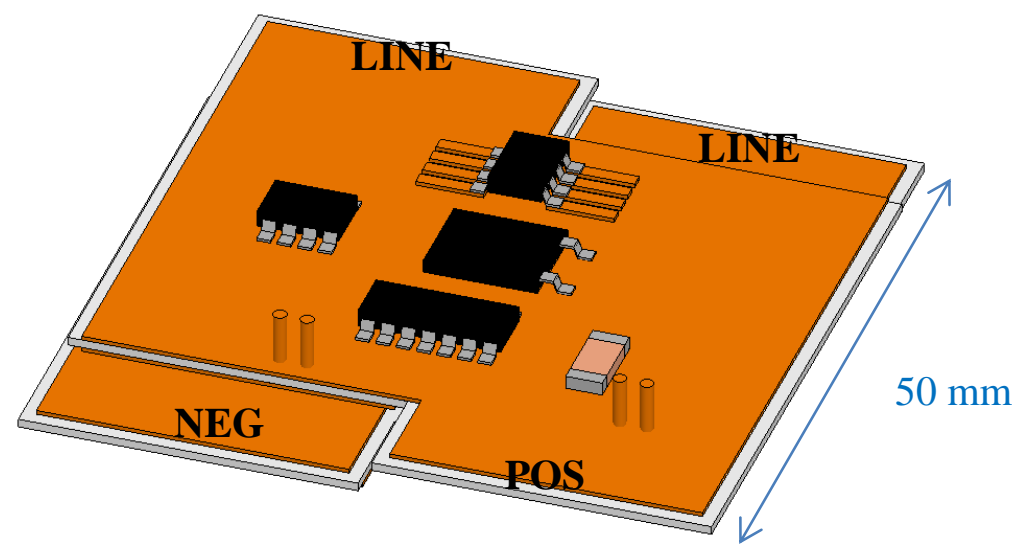

Figure 28. Packaging process of the planar power module

Table II. Dimensions of the Planar Power Module

\begin{tabular}{|c|c|}
\hline Material & Thickness (um) \\
\hline Copper (DBC) & 0305 \\
\hline Alumina (DBC) & 0.625 \\
\hline Die Attach Layer & 50 \\
\hline Spacer & $1200 / 1000$ \\
\hline IGBT & 0.14 \\
\hline Diode & 0.35 \\
\hline
\end{tabular}

\subsection{Simulation Model for Finite Element Analysis}

The Ansoft Maxwell 15 was used for the simulation. Figure 29 shows the simulation model with real dimensioned components. As explained earlier, the GMR sensor was placed between two current paths, and the dimension of the GMR sensor was determined based on a datasheet of a GMR sensor. The actual sensing element of the sensor has height bias in the package as $0.89 \mathrm{~mm}$ from the bottom surface of lead, so the sensing plane of the simulation model was also followed this dimension. All the observed magnetic-flux in this chapter will be the measurement result from this sensing element. The mesh is one of the most important factors to determine the accuracy of the simulation. 
In case of the AC magnetic-flux simulation, the skin effect does important role. Therefore, the minimum size of mesh was controlled based on the skin depth that can be defined as,

$$
\delta=\sqrt{\frac{\rho}{\pi \mu f}}
$$

For a copper conductor, the permeability $\mu$ is equal to $\mu_{0}$, and the resistivity $\rho$ is given $2.3 \cdot 10^{-6} \Omega-\mathrm{cm}$ at $100^{\circ} \mathrm{C}$.

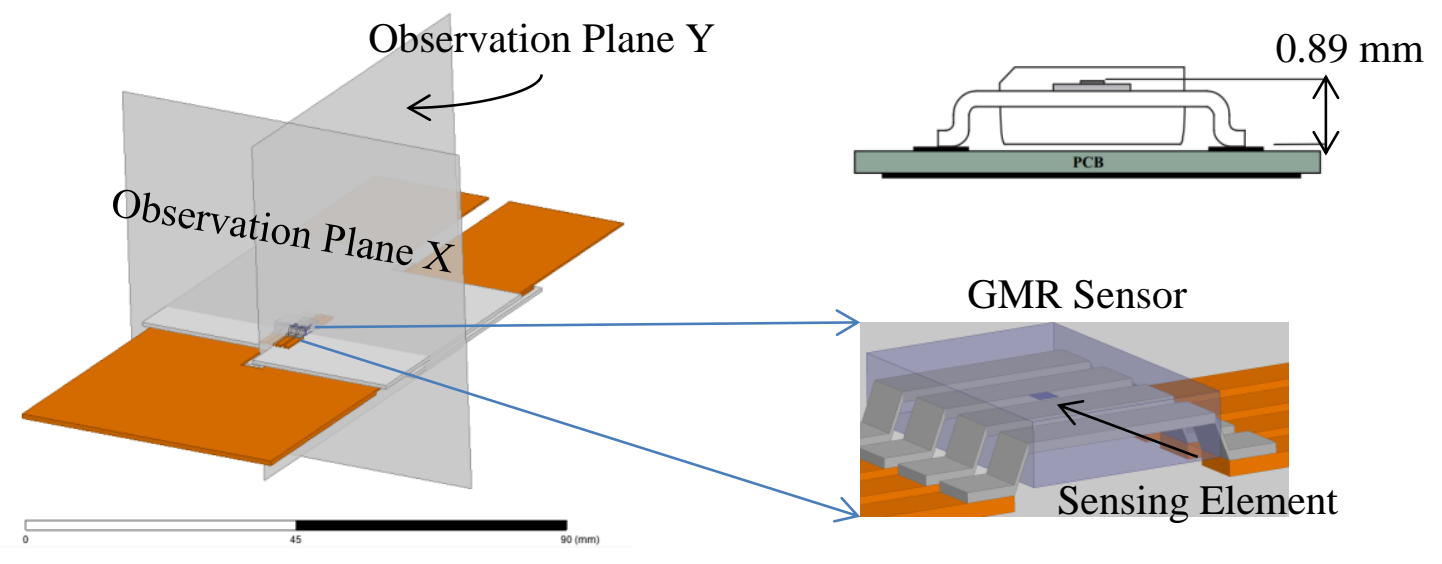

Figure 29. FEM Simulation model for flux density analysis

\subsection{Analysis of the Magnetic-flux Distribution}

The analysis of a magnetic-flux distribution of the planar power module is critical for the accurate measurement. The location of the GMR sensor should be chosen where can minimize the influence of eddy current effect so that magnetic-flux distribution can as consistent as possible for varying frequency. There were previous works that using GMR sensor as current-sensing method for low current applications [5, 9, 10]. However, the width of the current carrying conductors was narrow, so the eddy current effect did not give significant influence to the magnetic-flux distribution. The developing planar power module contains IGBT dice which can carry up to 150A. The large sized dice result 
wide $22 \mathrm{~mm}$ width copper trace, and the eddy current effect by switching frequency results non uniform magnetic-flux distribution around the wide copper trace. The simulation model was built to analyze the magnetic-flux density change by sweeping the frequency of the applied current. Figure 29(a) shows the simulation model for Ansoft Maxwell. To simplify the simulation mode, copper traces were left for current excitation, and the alumina of top DBC was left to emulate the actual magnetic-flux detect point. Since the GMR resistors are packaged in a SOIC8-lead type package, the actual magnetic-flux sensing point is middle of the packaged chip. Figure 29(b) shows the lay-up of the packaged GMR sensor. The simulated magnetic-field was monitored at the sensing element in the picture.

The applied current was $150 \mathrm{~A}$ with frequency swept from $1 \mathrm{~Hz}$ to $100 \mathrm{kHz}$, and Figure 30 shows the simulation result. As expected, as frequency increases the current is being concentrated at the edge of the current carrying conductor. The magnetic-flux density changes were measured at various locations to find the constant magnetic-flux region. Figure 30 shows the simulation result of the single copper trace which is in the dashed red box at Figure 29. The magnetic-field variation was normalized with respect to the 1 $\mathrm{Hz}$ simulation result. The region 1 and 3 were most influenced by eddy effect, and the magnetic-flux variation was $34 \%$ and $40 \%$. The region 2 and 4 showed relatively less variations which were $21.6 \%$ and $16.6 \%$ so these two locations with low variation were advantageous to locate GMR sensor. Each copper trace in the module contains one IGBT and diode in parallel, so in principle of electrical operation, both conductors will never conduct at the same time. 


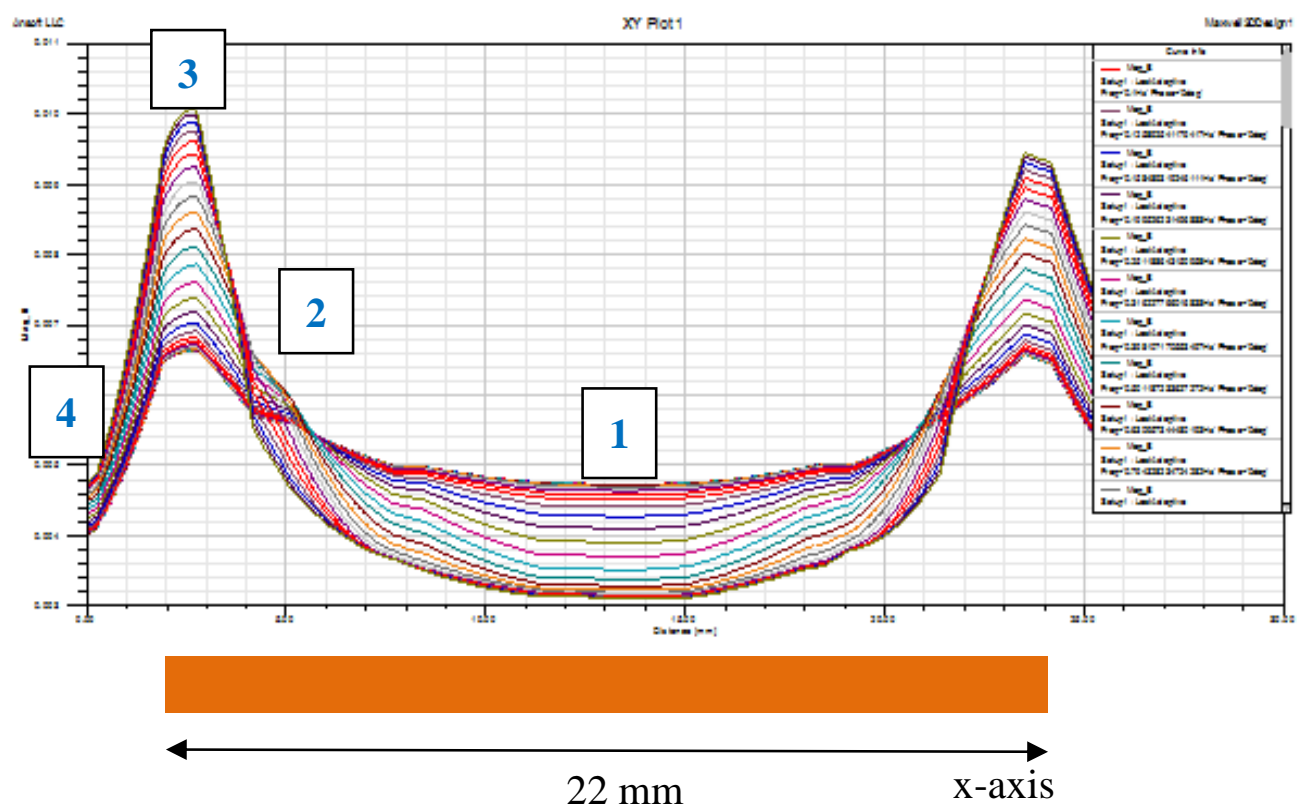

Figure 30. Magnetic-flux distribution on the copper trace with frequency sweep from $1 \mathrm{~Hz}$ to $100 \mathrm{kHz}$

Therefore it can be assumed that the other copper trace at the left side will have the same magnetic-flux distribution as mirrored image. The region 4 is between two copper traces, and this spot will have same magnetic-flux distribution for both current conduction cases. Also by sensing the magnetic-flux at this region, only one GMR sensor can be used for sensing the current at both copper traces, which will save number of components for signal-conditioning circuit.

\subsection{Influence of the Frequency and Amplitude to Magnetic-field Distribution}

\subsubsection{The harmonic frequency analysis of the magnetic-flux density}

The targeting application of the planar module is the automotive motor drive. The conventional switching frequency of the motor drive is around $10 \mathrm{kHz}$, but the non-sinusoidal load current will contain harmonics and the current sensor should have enough bandwidth to capture harmonic frequencies as well. To evaluate the harmonic 
frequencies of the load current, Maxwell transient simulation model was built. Figure 31(a) shows the simulation model that built for transient simulation, and Figure 31(b) shows the current excitation to the current paths.

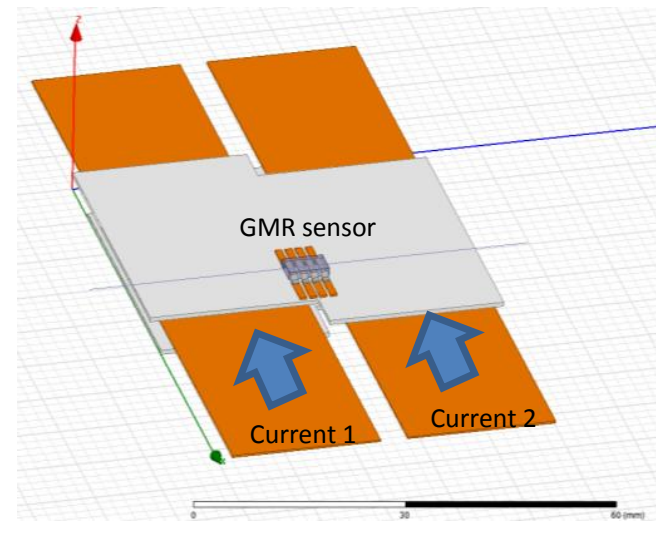

(a)

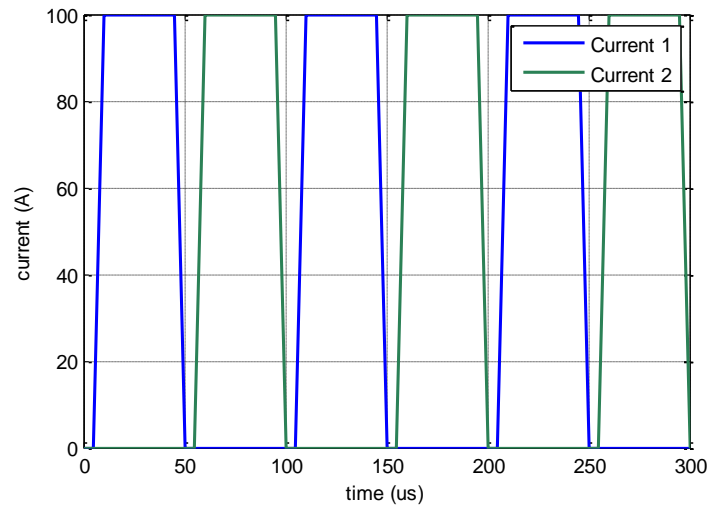

(b)

Figure 31. Transient simulation model. (a) Simulation model. (b) Current excitation to the copper bars.

Two square wave currents were applied complimentary with same duty and amplitude. The magnetic-flux density at the middle of between two paths was observed to see the frequency response for alternating current excitations.

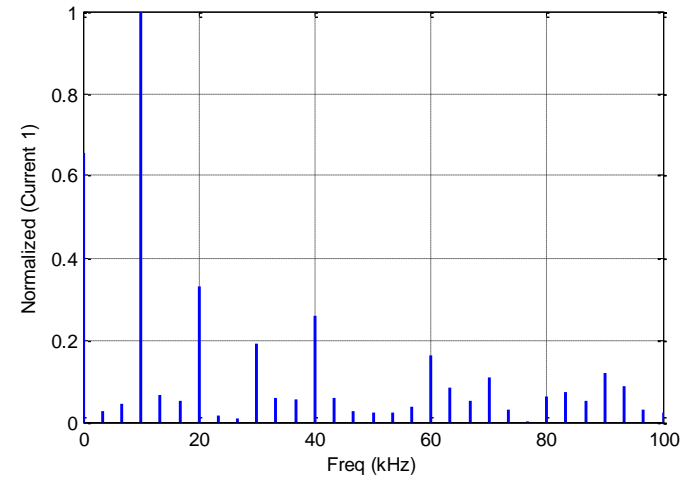

(a)

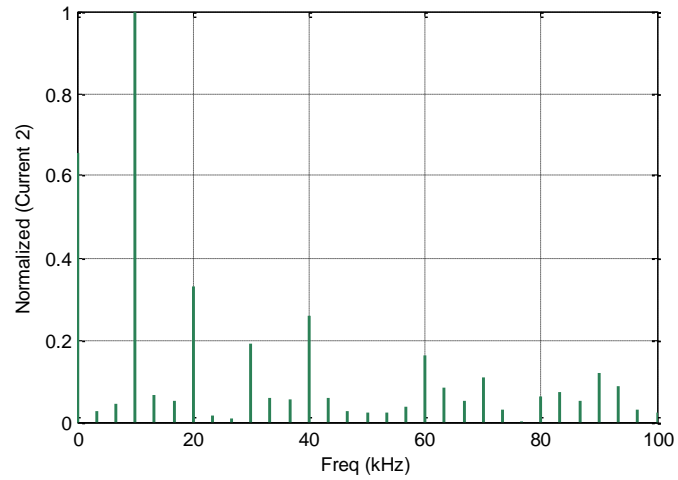

(b)

Figure 32. FFT of two current excitations (a). FFT of current 1. (b) FFT of current 2. 
The Fast Fourier Transform (FFT) analysis was done for applied currents to see the frequency components. Both square wave currents had same magnitude, frequency, and duty so the FFT analysis result was identical. The fundamental frequency was found as $10 \mathrm{kHz}$, and the amplitude decreased below $10 \%$ after $100 \mathrm{kHz}$. After running the simulation, the magnetic-flux density change versus time was plotted, and the FFT analysis was performed in the same way.

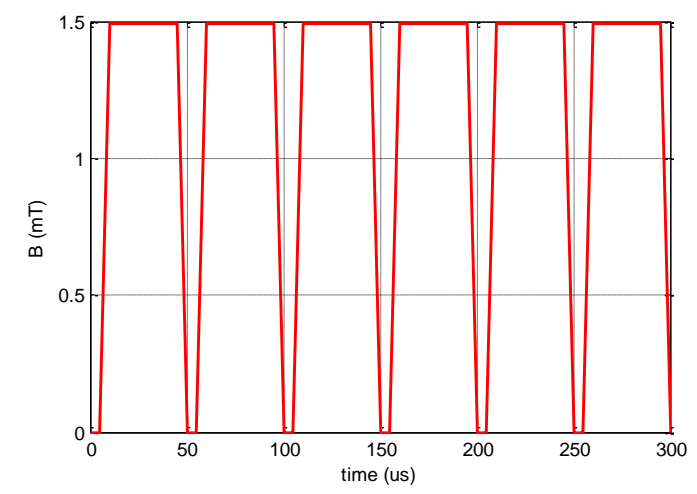

(a)

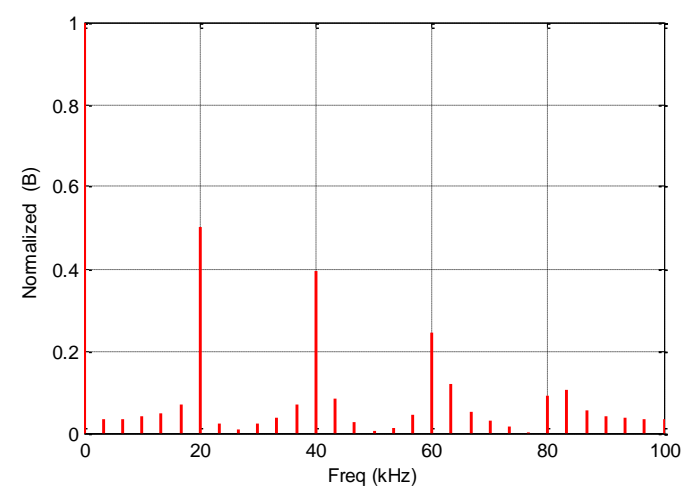

(b)

Figure 33. Observed magnetic-flux density in the middle of current paths. (a) Magnetic-flux density vs. time. (b) FFT analysis of the magnetic-flux density.

Because the sensing point was the middle of two current paths, the observed magnetic-flux density combined fluxes came from both conductors. Since the magnetic-flux density taking both currents signals, the fundamental frequency was doubled as $20 \mathrm{kHz}$, and the amplitude dropped below $10 \%$ at fifth harmonic which was $100 \mathrm{kHz}$. Therefore, if the GMR senor has a bandwidth can capture up to $100 \mathrm{kHz}$, it can be used for the automotive motor drivers. The previous study [6] showed that the GMR sensor has $-1 \mathrm{~dB}$ bandwidth at $400 \mathrm{kHz}$, so this bandwidth range should be fine to cover most of harmonic frequencies. 


\subsubsection{The influence of the Eddy effect to the measurement}

The magnetic-flux density seen by the GMR sensor will be influenced by frequency. Even though the motor drive application will switch at $10 \mathrm{kHz}$ frequency, the sensor should have constant measurement over wider frequency that can capture the harmonic frequencies. From the previous paragraph, the harmonic frequency of the magnetic-field density dropped to $10 \%$ at $100 \mathrm{kHz}$. So the influence of the eddy effect was analyzed from $10 \mathrm{kHz}$ to the $100 \mathrm{kHz}$. As switching frequency increases, the skin depth gets smaller and the current density at the edge of current path gets higher. The magnetic-flux density seen by the GMR sensor increased linearly as frequency increased by $2.67 \%$.

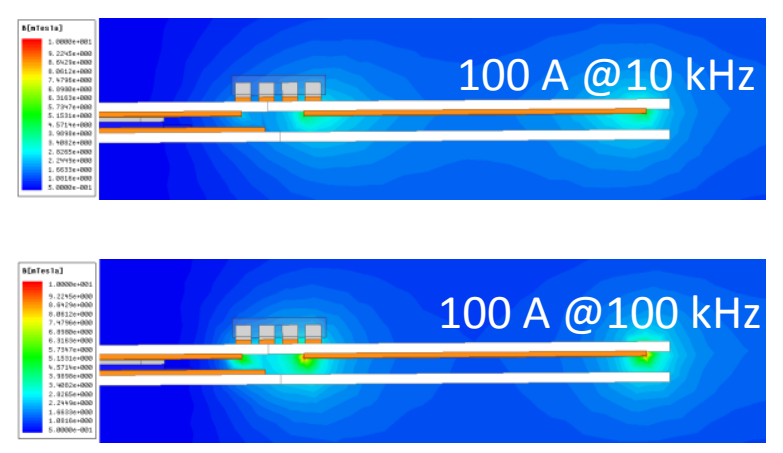

(a)

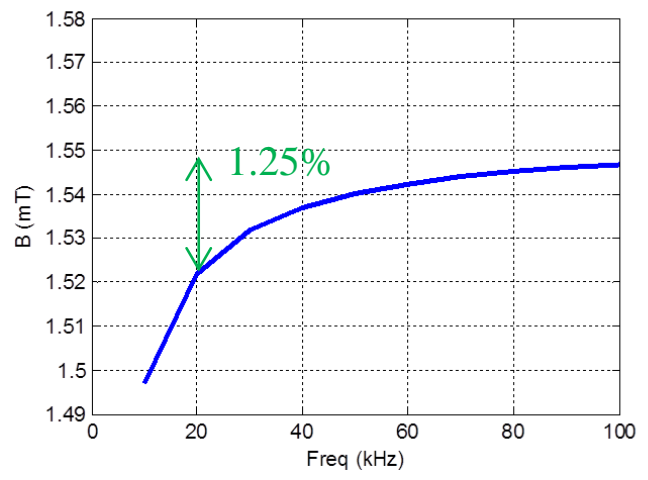

(b)

Figure 34. Simulation result with various frequency conditions. (a) Flux distribution on the observation plane X. (b) Magnetic-flux density versus frequency.

The magnetic-flux distribution on the observation plane $\mathrm{X}$ was studied so far, and the flux distribution on the $\mathrm{Y}$ observation plan also should be analyzed. The current conduction path by inverter operation for one set of IGBT and diode is shown in Figure 35. One conduction path is from DC power supply to the line output through the IGBT, and the other conduction path is the freewheeling stage through the parallel diode. Since 
IGBT requires extra connection to the gate and source for driving, the top of the IGBT should be reserved for gate driver IC and miscellaneous devices around of it. Remaining spots for GMR sensor are between IGBT and diode, and on top of the diode. In case of location between two devices, there is a height difference from conduction path for two current conduction cases. Therefore, the GMR sensor was decided to be placed on the top of the diode on y-axis.
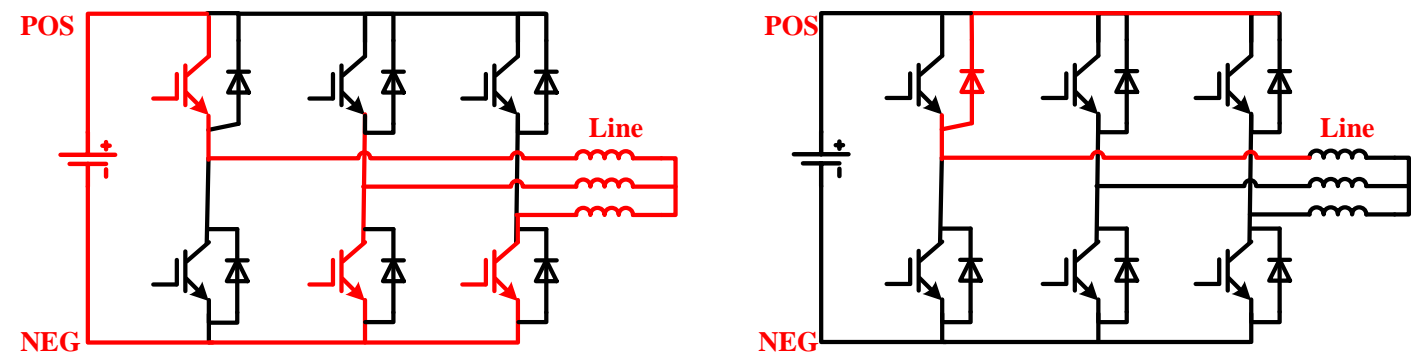

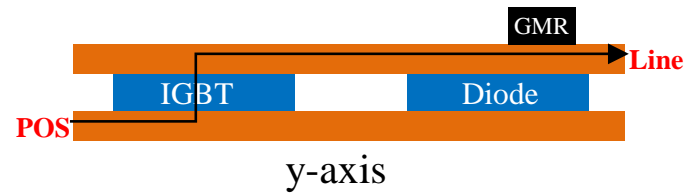

(a)

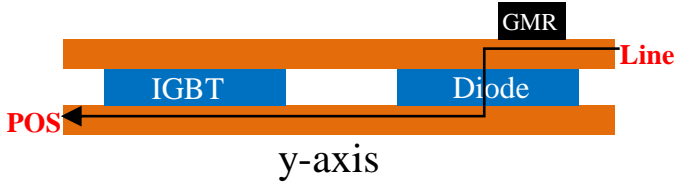

(b)

Figure 35. Current conduction paths of planar power module

\subsubsection{The influence of the Alignment of the Sensor}

To see the influence of the misalignment of the GMR sensor in a packaging level, the magnetic-flux density at different locations were observed. The Point 1 is the exact position that was observed for previous simulations, and Point 2 and Point 3 are the biased points that are $3 \mathrm{~mm}$ away to left and right sides. The current excitations to two current paths were kept same, and magnetic-flux densities at two additional points were observed. 


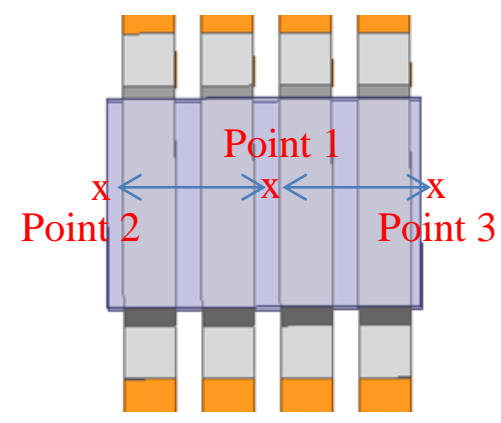

Figure 36. Magnetic-flux density observation at biased locations.

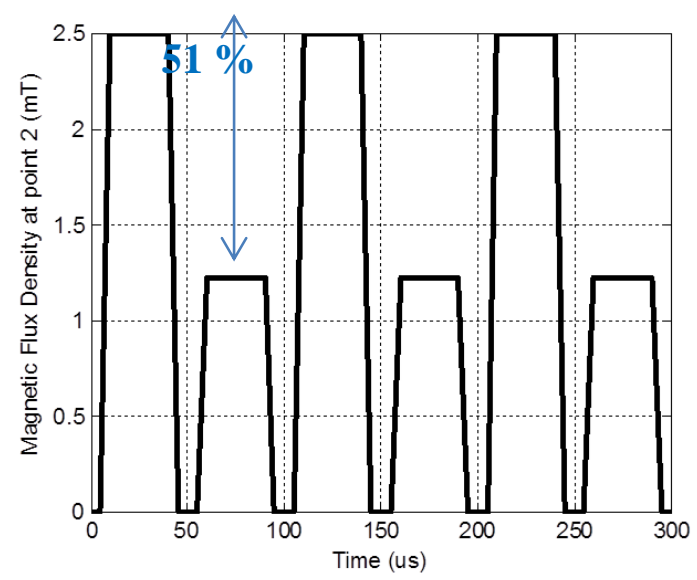

(a)

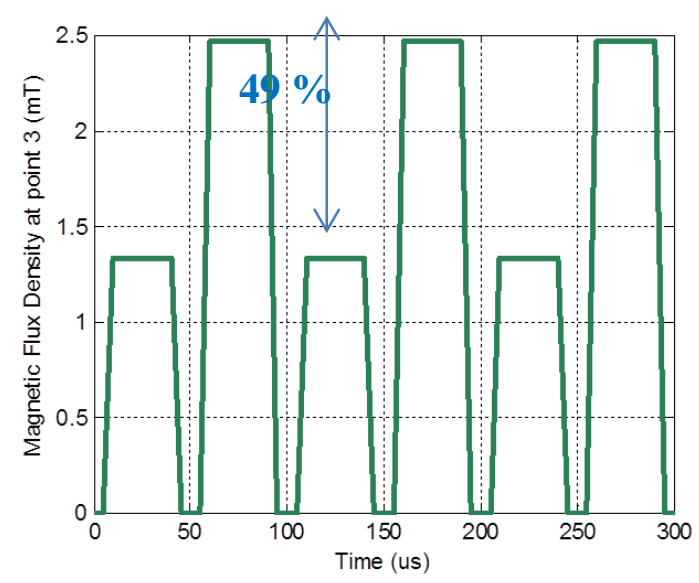

(b)

Figure 37. Magnetic-flux density at two biased points; (a) Point 2. (b) Point 3.

Figure 37 shows measured magnetic-flux densities that observed at two additional points. Both simulation results showed that the magnetic-flux that measured from one current excitation was biased by $50 \%$ from the other current excitation. This simulation result implies that the accurate alignment in a packaging level is critical for the accurate measurement.

\subsection{Conclusion}

The influence of the sensing position and frequency of current to the GMR sensor was studied in this chapter. Since the magnetic-flux density seen by the GMR sensor is 
directly influenced by sensing distance and eddy effect resulted by frequency, the FEA simulation was used to predict the difference. Based on this simulation, the GMR sensor is able to sense from $65 \mathrm{~A}$ to $200 \mathrm{~A}$. This sensing range is enough to use GMR sensor as over-current protection purpose, but not enough for control purpose. In the future, to maximize the functionality of the GMR sensor in the module, the geometry of the sensor and module need to be modified.

From the previous paragraph, the harmonic frequency of the magnetic-field density dropped to $10 \%$ at $100 \mathrm{kHz}$. The influence of eddy-current effect was analyzed from 20 $\mathrm{kHz}$ to the $100 \mathrm{kHz}$. As switching frequency increases, the skin depth becomes smaller and the current density at the edge of current path becomes higher. The magnetic-flux density seen by the GMR sensor increases linearly as frequency increases, and the magnetic-flux density increases by $1.25 \%$ from $20 \mathrm{kHz}$ to $100 \mathrm{kHz}$. Therefore, by placing the GMR sensor at that location, $1.25 \%$ error by frequency change should be expected. 


\section{Chapter 4. INTEgRATION OF THE GMR SENSOR INTO THE Planar Power Module}

The magnetic-flux distribution of the module was studied in the previous chapter, and based on the study, the GMR sensor should be able to sense the current flowing through the bottom copper trace of the DBC substrate. The same test board that fabricated for the thermal analysis was used for the electrical test. Since the module was still under the developing stage, the additional test method was required, and the double pulse tester was chosen as a test method. The double pulse tester circuit has a simple operating principle, but can test a wide range of current and switching transient conditions. The test module was connected in series with a winding to inductor so that the GMR sensor can sense the inductor current. The measured signal by the GMR sensor was compared with high bandwidth current probe for comparison. The preliminary measurement result from the GMR sensor showed very low SNR which is hard for practical use. The signal-conditioning circuit was designed to filter out the noise, and the out of the signal-conditioning circuit showed consistent measurement result with current probe. The over-current protection circuit was designed using comparator and shut down function of the gate driver. The over-current protection circuit could shut down the system as designed, when the over-current is detected.

\subsection{The Test Setup of the GMR Sensor-Double Pulse Tester}

The GMR sensor can detect not only constant field, but also time varying field. As long as the sensing field is in a linear measurement range of the sensor, any current within a bandwidth can be detected. The double pulse tester has several characteristics that can test the measurement capability of the GMR sensor. During the initial charging time, the low frequency response of the sensor can be tested, and during the switching 
transients, the high frequency dynamic performance of the sensor can be tested. Also the double pulse tester will show the hard switching behavior which will result common mode noise problem. This paragraph will show the test setup and baseline measurement that has problems to be solved. Figure 38is the built double pulse tester. It consists of input capacitor, decoupling capacitor, IGBT half bridge module, gate driver circuit, and inductor. Table III shows the part number and rating of each component.

Table III. Component list of the Double pulse tester

\begin{tabular}{|c|c|c|}
\hline Part Name & Part Number & Rating \\
\hline Input Capacitor & 8D09AT & $8200 \mathrm{uF} / 450 \mathrm{VDC}$ \\
\hline Decoupling Capacitor & SCD105K601A3Z25 & $1 \mathrm{uF} / 600 \mathrm{VDC}$ \\
\hline IGBT Module & CM200DY-24H & $1200 \mathrm{~V} / 200 \mathrm{~A}$ \\
\hline Gate Driver & Powerex BG2C & Vout: +15V/-8V \\
& & Source/sink current: $3 \mathrm{~A}$ \\
\hline Inductor & Iron Powder & $700 \mathrm{uH} / 20 \mathrm{pF} / 2.1 \Omega$ \\
\hline
\end{tabular}




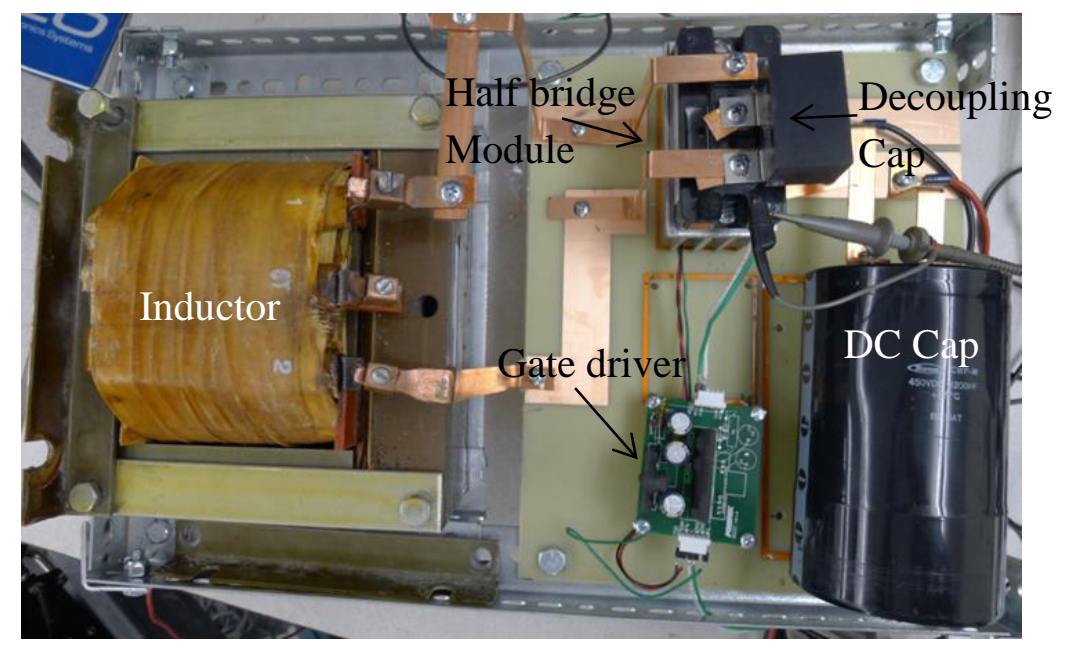

Figure 38. Double pulse tester setup

The copper bar was used to make connection between inductor and IGBT module, and the connection was intentionally designed to have a large loop so that the GMR sensor test board can be connected in series with a copper bar. In Figure 39, during the period I, the low side IGBT conducts and the inductor current linearly increases with respect to the input voltage (Figure 40(a)). During the period II, the low side IGBT turns off and the inductor current starts freewheeling through the high side diode. The inductor current decreases linearly during this period, and when the gate signal is applied again to the IGBT, the inductor current linearly increases again. During this transient, however, the diode does not instantaneously turns off so the devices will experience shoot through during this transient. It will results large current spike and the resulting magnetic-field will be sensed my GMR sensor as normal current signal. This kind of signal is not an actual fault case, but GMR sensor will consider this spike as a fault case, and can cause malfunction of the over-current protection circuit. 


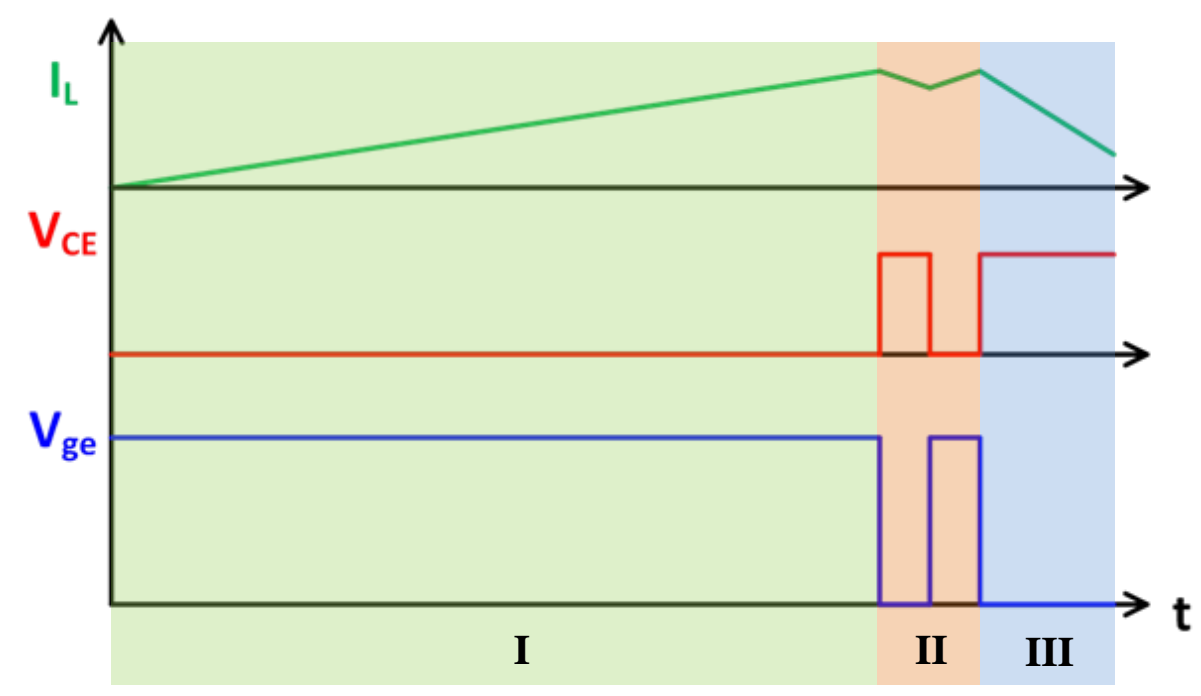

Figure 39. Waveform illustration of the double pulse tester. From Top: Inductor current, collector-emitter voltage, and gate voltage.
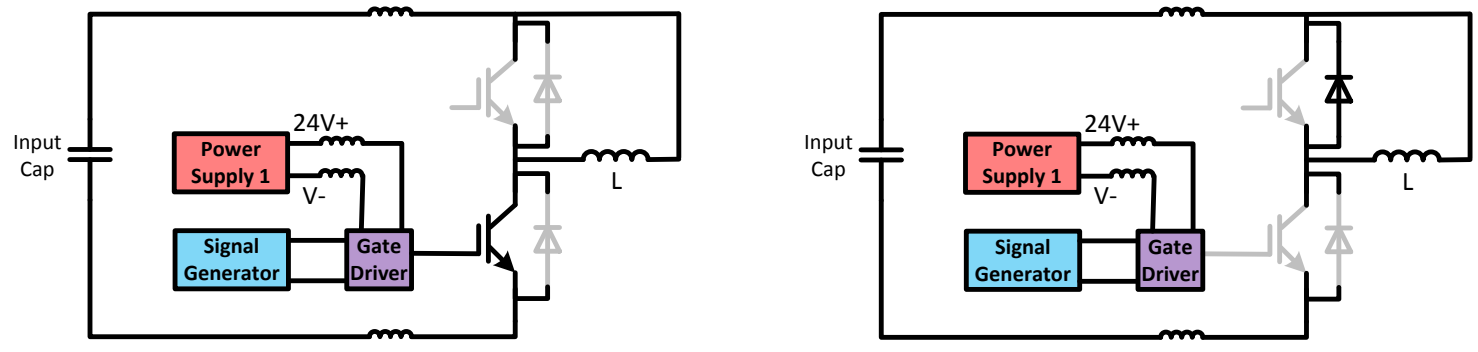

Figure 40. Switching sequence of the double pulse tester. (a) Low IGBT conducts and inductor current builds up. (b) IGBT turns off and inductor current freewheels through high side diode 


\subsection{Preliminary Measurement of the GMR Sensor}

The GMR sensor was packaged on top of the DBC substrate which has same dimension as actual power module. Copper layers at both top and bottom layers were patterned by wet etching process, and bottom side copper was patterned as an actual copper trace width. The bottom copper trace then attached to copper strip, and the copper strip was connected in series with the inductor winding of the double pulse tester. So when the double pulse tester operates, the inductor current will go through the copper trace under DBC substrate which can emulate the operation of the planar module. With

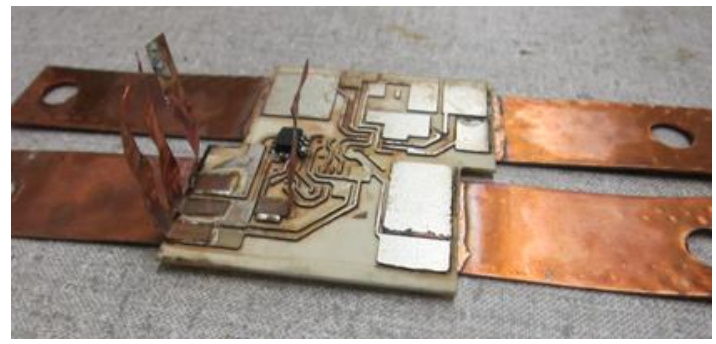

Figure 41. The preliminary GMR sensor test board. $15 \mathrm{~V}$ input voltage to the GMR sensor, the output voltage of the GMR sensor was measured by an oscilloscope. Figure 42 shows the output voltage waveform of the GMR sensor. The measured signal shows high frequency intrinsic noise of the GMR sensor in an overall measurement, and more importantly, at the IGBT turn on transient huge spike happens which will result malfunction of the over-current protection circuit. During the switching transient, because of the reverse recovery time of the diode, both diode and IGBT turns on at the same time. This will result shoot though for a short moment, and it will result high current spike. This current spike will not damage the module, but GMR sensor has a high enough bandwidth to detect this spike, and it will shut down the system unnecessarily. The over-current protection circuit will finally compare the measured signal to the reference voltage, but the intrinsic noise of GMR sensor and current overshoot can result wide range of error. Therefore, the signal-conditioning circuit that can attenuate measurement noise had to be designed. 


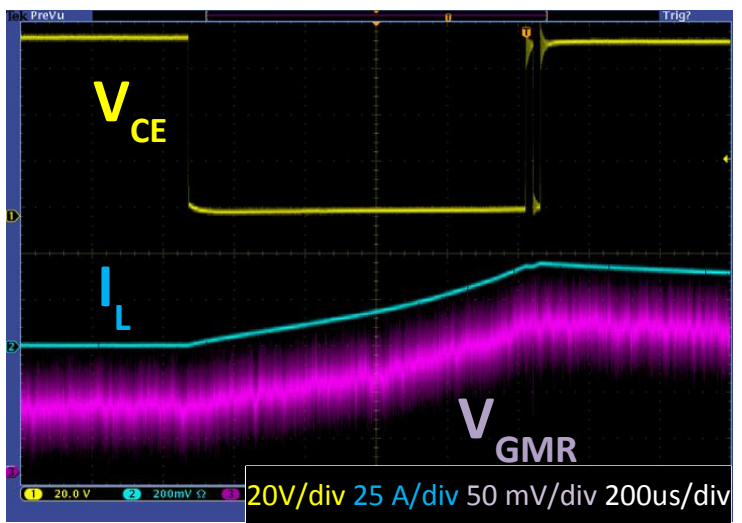

(a)

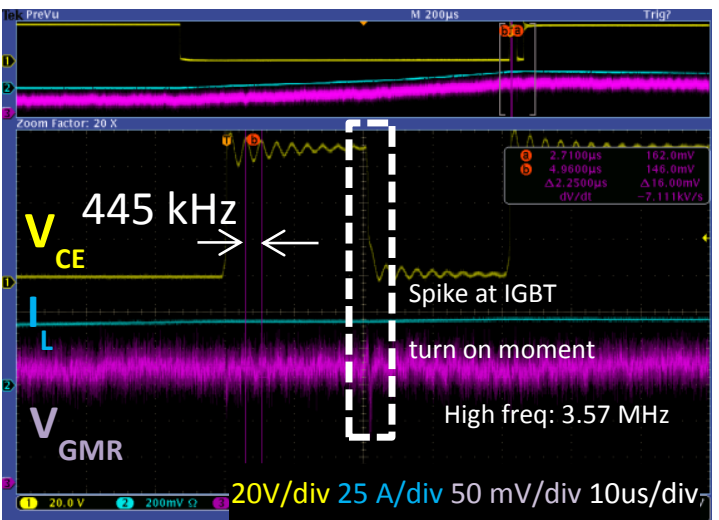

(b)

Figure 42. The output voltage waveform of the GMR sensor. (a) Measured signal. (b) Zoom in the switching transient

\subsection{Design of the Signal-conditioning Circuit}

The output voltage waveform of the GMR sensor in Figure 42 showed three major noise sources. The first noise was the intrinsic noise coming from the GMR sensor. This noise happened regardless the magnetic-field was applied or not, but this noise has relatively high ratio to the signal so its amplitude should be attenuated. The second noise was the common mode noise that follows the oscillation frequency of the collector-emitter voltage of the IGBT. Since the ground of the power device, gate driver, and GMR sensors were tied to a common ground, the noise happened at the voltage signal directly influenced the output voltage of the GMR sensor. The third noise was the big spike during the IGBT turn on transient. This spike is an actual current signal that measured by GMR sensor, but this spike also had to be attenuated to avoid malfunction of the over-current protection circuit. Looking back the frequency components of each noise signal, the intrinsic noise of the GMR sensor had $3.57 \mathrm{MHz}$, and the current spike was estimated as $3.33 \mathrm{MHz}$ based on the reverse recovery time of the diode (300 ns). The high frequency signal as this noise can be easily attenuated by using proper operational amplifiers. However, the low frequency oscillation that caused by common-mode noise 
cannot be attenuated by the operational amplifier. To attenuate common-mode signal, the differential amplifiers are preferred, because these amplifiers amplifies differential signals as usual amplifier, but amplifies the common-mode signal with unity gain. However, the input resistance of the differential amplifier has low resistance limitation to avoid low input impedance. Therefore, the instrumentation amplifier that has buffers at the input side of the differential amplifier was chosen for a signal conditioning.

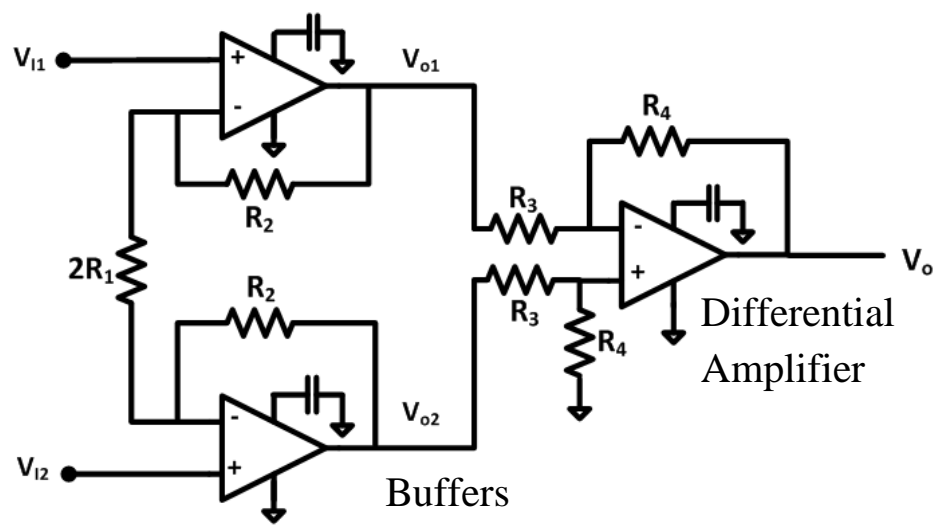

Figure 43. Schematic of the instrumentation amplifier.

Figure 43 shows the schematic of the instrumentation amplifier that will be used for signal conditioning. Two buffers at the input side provide high input impedance to the differential amplifier, and also work as non-inverting amplifier. The gain of two buffers should be kept identical and the gain of two buffers can be expressed as,

$V_{o 1}=V_{I 1}\left(1+\frac{R_{2}}{R_{1}}\right) \quad$ and $\quad V_{o 2}=V_{I 2}\left(1+\frac{R_{2}}{R_{1}}\right)$

The output voltages from buffers are connected to the differential inputs of the differential amplifier. To maximize the common-mode noise rejection ratio, four resistances are kept identical. The output voltage of the differential amplifier follows the equation,

$V_{o}=\left(\frac{R_{3}+R_{4}}{R_{3}}\right) \cdot\left(\frac{R_{4}}{R_{3}+R_{4}}\right) V_{o 2}-\frac{R_{4}}{R_{3}} V_{o 1}$ 
If four resistances are same, the output voltage simply becomes the difference between two input voltages.

$$
V_{o}=V_{o 2}-V_{o 1}
$$

The commercial instrumentation amplifier IC INA333 from Texas Instrument was used for this study to maximize the performance of the signal-conditioning circuit. Commercial IC has on-chip resistors that are laser etched for accurate and consistent resistance, and only one resistor is left for gain control which is $2 R_{1}$ term in Figure 43. The frequency response of the INA333 is shown in Figure 44. The gain was set as 10 for the experiment and the $-3 \mathrm{~dB}$ bandwidth with this gain is $35 \mathrm{kHz}$. From the gain curve in Figure 44, the gain of the amplifier goes well below $-40 \mathrm{~dB}$ after $1 \mathrm{MHz}$, so the high frequency noise that observed in preliminary measurement can be well attenuated. Also the common-mode rejection ratio is $110 \mathrm{~dB}$ when the gain is 10 . This rejection ratio should be high enough to eliminate the common-mode noise that observed in a preliminary measurement.

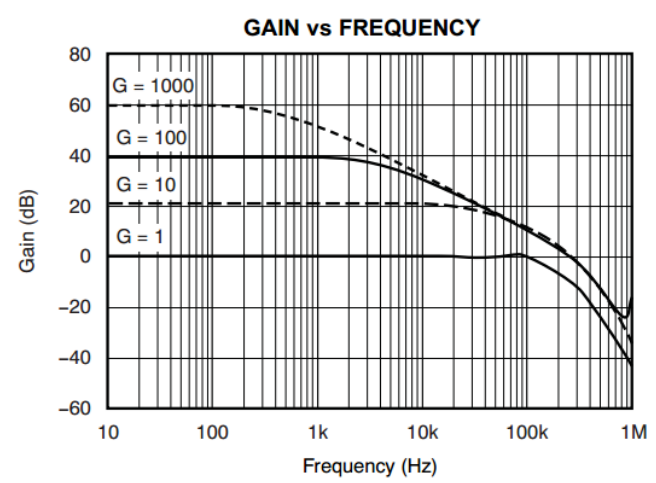

(a)

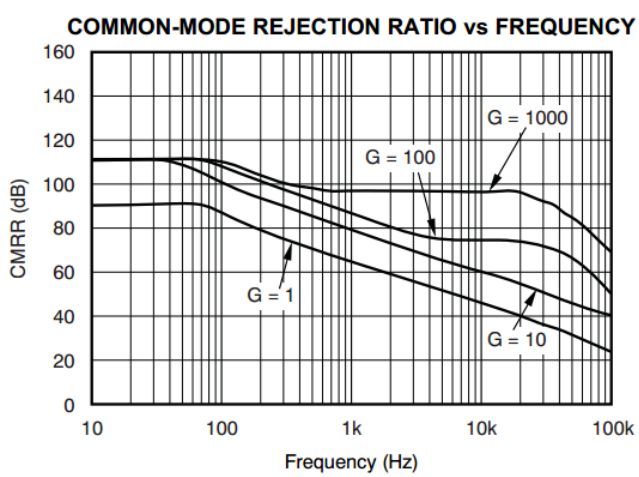

(b)

Figure 44. Frequency response of the INA333 instrumentation amplifier. (a) Gain curve. (b) CMRR curve.

The test circuit with instrumentation amplifier was fabricated on a PCB. Figure 45(a) shows the schematic of the signal-conditioning circuit. The positive and negative outputs were connected to the differential input of the instrumentation amplifier, and the gain of 
the instrumentation amplifier was set to 10 . Figure 45(b) shows the fabricated test circuit. The test circuit was fabricated by wet etching process, and headers were added for jumping to the DBC test board that has the GMR sensor and also to use as test points.

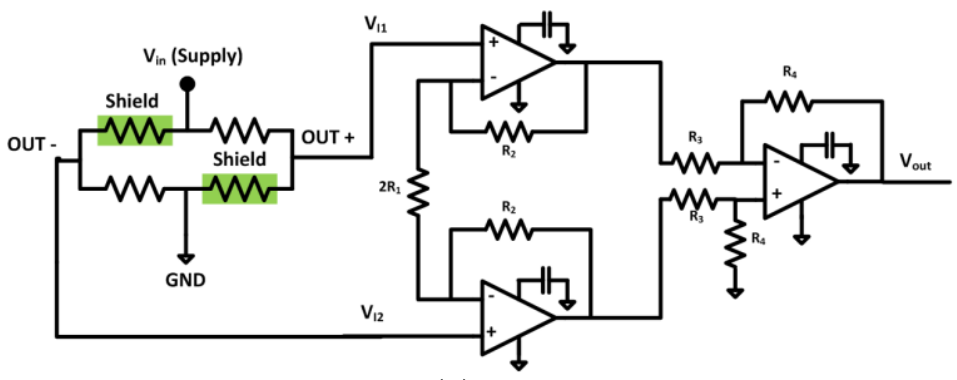

(a)

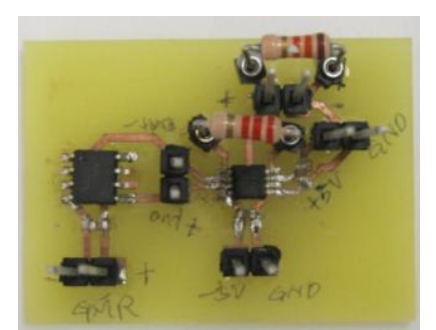

(b)

Figure 45. Fabricated signal-conditioning circuit. (a) Schematic of the signal-conditioning circuit. (b) Fabricated board on a PCB.

Figure 45 shows the measured waveform of the signal-conditioning circuit. The blue waveform is the inductor current that measured by high bandwidth current probe, and the purple waveform is the output of the signal-conditioning circuit. The high frequency noise came from the GMR sensor itself and current spike were well removed, and the common-mode noise oscillation was also removed. There is little oscillation in a zoomed in waveform, but that oscillation is coming from the settling time of the instrumentation amplifier. There is one thing needs to be pointed out that the measured waveform by current probe and GMR sensor has little discrepancy at low current measurement. This is because of the low magnetic-field density seen by the GMR sensor at low current. The magnetic-flux density was simulated in Chapter 3, and the magnetic-flux density goes below linear sensing range of the GMR sensor after below $65 \mathrm{~A}$. This is acceptable for over-current protection circuit, because just one point needs to be accurately sensed for over-current protection, but to use the GMR sensor for actual control purpose, the sensing 
location of the GMR sensor should modified in the future. Overall, the measured inductor current by the GMR sensor showed consistent measurement result with the measurement done by high bandwidth current probe.

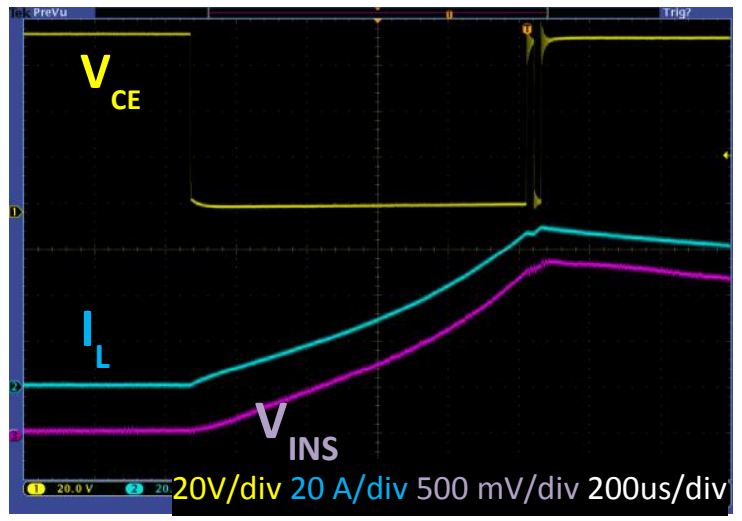

(a)

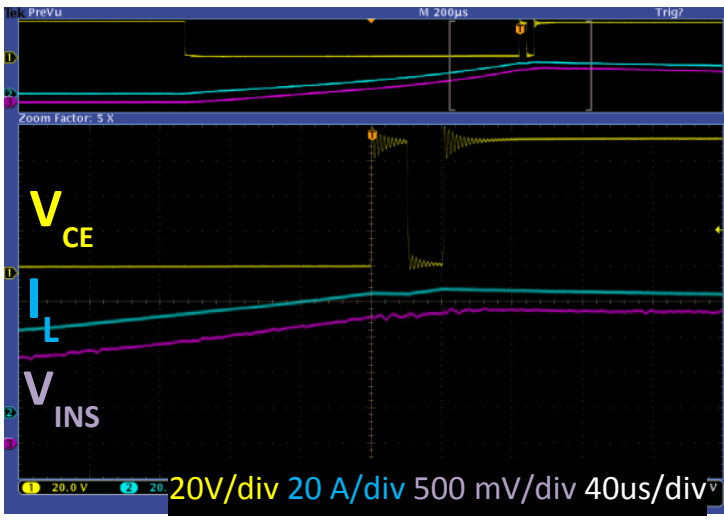

(b)

Figure 46. Output of the signal-conditioning circuit. (a) Comparison with the current probe. (b) Zoomed in picture of transient moment.

\subsection{Design of the Over-current Protection Circuit}

The over-current protection circuit was designed using the measured current by the GMR sensor. The shunt down pin of the gate driver circuit was used to configure the over-current protection circuit. The gate driver from International Rectifier IR21844 was used as gate driver. When the voltage at $\mathrm{V}_{\mathrm{SD}}$ is higher than threshold voltage, the gate driver will work as normal, but when the voltage at $\mathrm{V}_{\mathrm{SD}}$ goes below threshold voltage, the gate driver will stop working. To make use of this function, the comparator was added between the signal-conditioning circuit and gate driver. The output of the signal-conditioning circuit was compared with reference voltage of the comparator. If the reference voltage is higher than input signal, the output voltage will be high, but once the input signal exceed the reference voltage, the output voltage of the comparator will go to 
low level and the gate driver will stop generating output signal. Figure 47 shows the waveform of the test board that fabricated to prove the concept. Figure 47(a) shows the normal operating condition. VSD was kept to the high level all the time, and the output signal of the gate driver was inversed square wave as expected. Figure 47(b) shows the situation with the over-current detection. The input signal to the gate driver and output signal of the gate driver operate normally at the beginning, but at one point, the VSD goes low level which means the over-current detection. At this transient, the input signal to the gate driver is normal, but the output of the gate driver goes zero until VSD goes back to high level. This experiment result shows that if the comparator can give proper VSD signal to the gate driver, the gate driver can stop applying gate signal to the IGBT. From this measurement, the time delay to shut down gate driver after over-current detection was measured as $240 \mathrm{~ns}$.

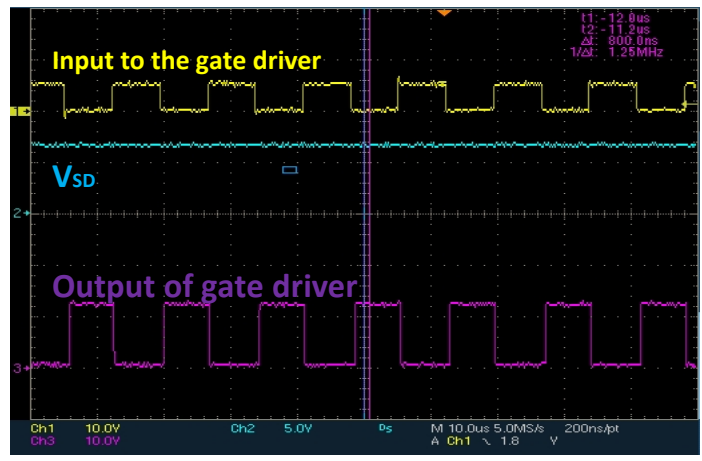

(a)

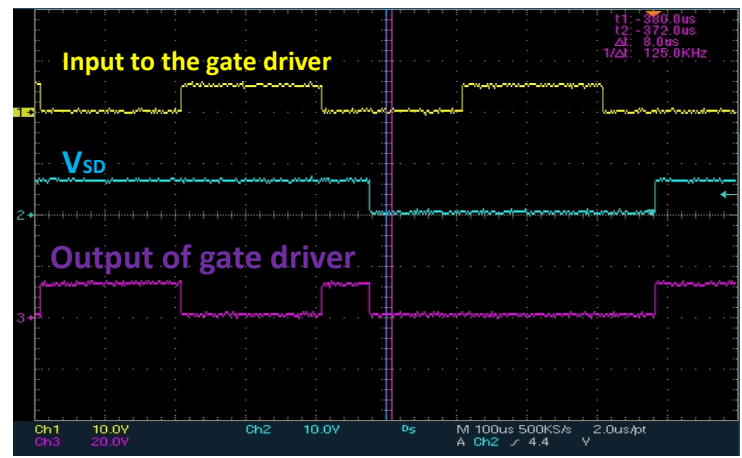

(b)

Figure 47. The waveform of the preliminary over-current protection circuit.

Figure 48 shows the schematic of the over-current protection circuit. The output of the signal-conditioning circuit was connected to the negative input of the comparator, and the reference voltage level was controlled by potentiometer as necessary. For this experiment, the inductor current was controlled to increase up to $75 \mathrm{~A}$, and the reference voltage to the comparator was controlled to shut down the gate driver at $45 \mathrm{~A}$. There were two 
reasons that chose this current level. The developed prototype planar module is under test with 40 A $\mathrm{I}_{\mathrm{CE}}$ current for now, and the three phase inverter that packaged with the prototype planar module will also operate with this current level. Therefore, the prototype board was also designed to consider $45 \mathrm{~A}$ as fault case. The second reason was if the current level is low, the magnetic-field density is also low. In that case, the SNR will become low compared with high current detecting case which can be considered as worst case. If the designed circuit works well with low current level, there should be no problem with high current operation, unless the magnetic-field saturates the GMR sensor.

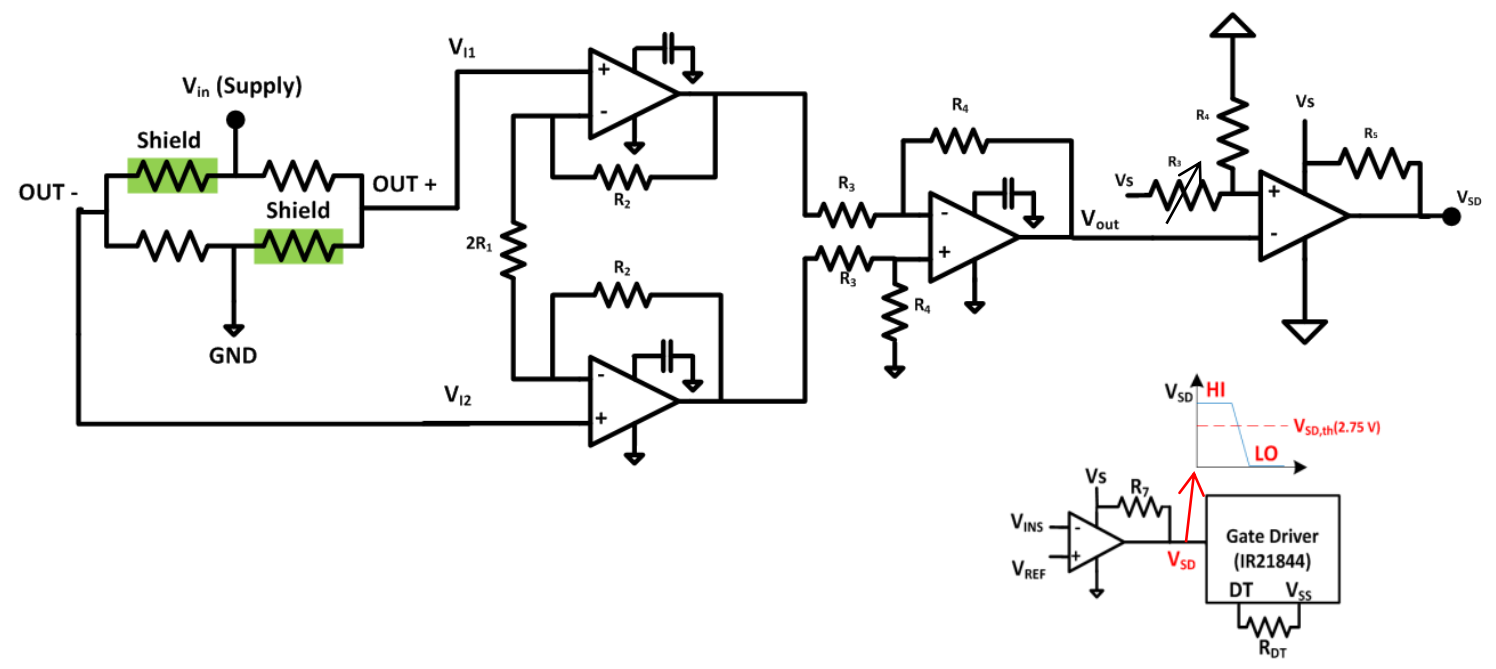

Figure 48. Schematic of the signal-conditioning circuit.

Figure 49(a) shows the fabricated test board that has same footprint as the top substrate of the planar module. The fabricated circuit has a GMR sensor, signal-conditioning circuit, comparator/amplifier, and dual channel gate driver circuit with bootstrap diode and capacitor.

Table IV. List of components of the gate driver circuit.

\begin{tabular}{|c|c|}
\hline Part Number & Description \\
\hline IR2184 (IR21844) & Dual channel gate driver (with dead time control) \\
\hline AA005 & GMR field detector \\
\hline
\end{tabular}




\begin{tabular}{|c|c|}
\hline LM392 & Comparator/Amplifier in single package \\
\hline INA333 & Instrumentation amplifier \\
\hline CSD01060E-ND & $600 \mathrm{~V}$ Bootstrap diode \\
\hline $445-7925-1-\mathrm{ND}$ & $250 \mathrm{~V} / 1 \mathrm{uF}$ Bootstrap capacitor \\
\hline $445-1314-1-\mathrm{ND}$ & $50 \mathrm{~V} / 0.1 \mathrm{uF}$ Decoupling capacitor \\
\hline
\end{tabular}

Figure 49(b) shows the measured waveform of the circuit. The purple curve shows the output of the signal-conditioning circuit, and the blue curve shows the reverence voltage set by a potentiometer. The reference voltage was set to consider the $45 \mathrm{~A}$ as over-current transient, and at that moment the $\mathrm{V}_{\mathrm{SD}}$ level goes low which is well below the threshold voltage of the $\mathrm{V}_{\mathrm{SD}}$ pin in a gate driver. Therefore, the designed circuit that contains current sensor, signal conditioning circuit, over-current protection circuit, and gate driver worked as designed. The layout of the integrated gate driver was packaged on the same size of top substrate of the planar module. The overall time delay from the current sense to system shut down was about 11 us. This time delay was mainly came from the signal conditioning circuit that contains three amplifiers. To further reduce the shut-down time, much faster instrumentation amplifier needs to be found. 


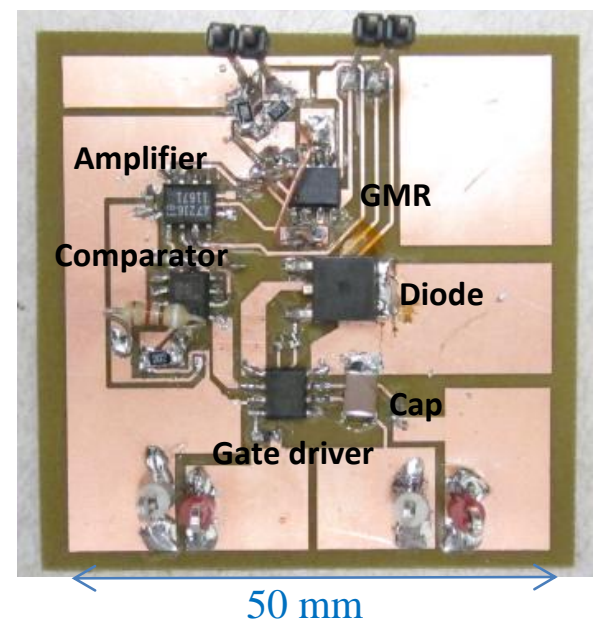

(a)

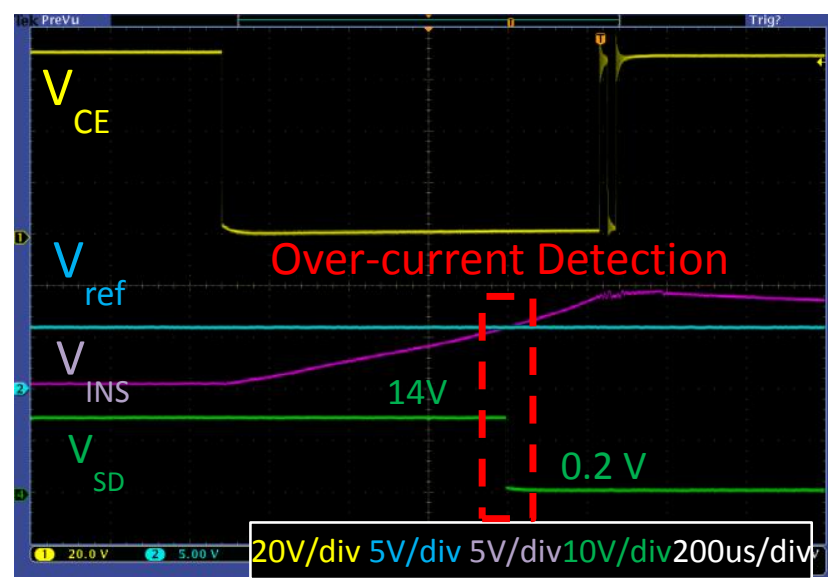

(b)

Figure 49. The complete gate driver board with over-current protection. (a) Test board. (b) Measured waveform with over-current situation.

\subsection{Conclusion}

In this chapter, the gate driver circuit with a current sensor, signal conditioning circuit, and over-current protection circuit were designed and tested by experiments. The output voltage of the GMR sensor that measured without any signal-conditioning circuit showed excessive noise that can cause malfunction of the over-current protection circuit. The signal-conditioning circuit with an instrumentation amplifier was designed, and most of noises were able to be attenuated with the designed signal-conditioning circuit. The over-current protection circuit was designed using a comparator and a shutdown function of the gate driver. The complete test board worked as designed, and the gate driver could be shutdown in 11 us. In the future, to further reduce the shut-down time, much faster instrumentation amplifier needs to be found. 


\section{Chapter 5. CONCLUSIONS AND FUTURE WORK}

\subsection{Main Contributions and Conclusions}

1. For the purpose of module integration, current sensors need to achieve the following key characteristics:

- High bandwidth

- High reliability

- Compact size

- Low measurement error in a wide range of temperatures

- Repeatable packaging process

- Low extra cost for integration

This study reviews many types of current-sensing methods, and key parameters for each sensing method are categorized as future reference. Most conventional current-sensing methods are shown to not be able to satisfy the required characteristics. Magnetic-flux sensing methods usually require a magnetic core for field concentration, and they are usually bulky and have low operating temperature range.

The GMR sensor was chosen as a solution for its many advantages that satisfy the key characteristics above. The GMR sensor is available in a standard IC package, which is very compact and enables a repeatable packaging process. It also can accommodate relatively high operating temperatures up to $150^{\circ} \mathrm{C}$, and its bandwidth overwhelms popular Hall-Effect sensors. However, the GMR provided contained excessive measurement noise, and a minimal change in the magnetic-flux density resulted in a large measurement error. Solving these problems is the main subject of this thesis. 
2. Like all other current-sensing methods, the GMR sensor is non-ideal for varying temperatures. The high junction temperature of the power devices influences the entire module's temperature, so quantifying the influence of temperature on the measurement accuracy is very important. The Wheatstone bridge helps to maintain good measurement capability over a wide range of temperatures, but the effect of a change in temperature on the GMR cannot be compensated by the unique structure of the sensor.

An experiment was performed with the input voltage and temperature as variables, while keeping the applied current constant. The output voltage was decreased as the temperature increased, which means the GMR effect got weaker as the temperature increased. The rate of change was analyzed, and the equation that shows the output voltage change according to temperature change was derived as a function of the input voltage. The base resistance change by temperature change was also studied using a constant current supply, and the resistance was linearly changed over wide temperature range. From this experiment, an equation was derived that shows the single resistance change over time.

3. The magnetic-flux density is the parameter that the GMR sensor measures, and this parameter is highly dependent on the sensing position and the frequency. FEA simulation was used to analyze the influence of the sensing position and frequency, and after this simulation the optimum location of the GMR sensor could be determined so the sensor can sense current flowing from both current traces.

4. The output voltage of the GMR sensor with the current excitation showed excessive noise that could result in the malfunction of the over-current protection circuit. The signal-conditioning circuit was made by an instrumentation amplifier and could attenuate most noises. The high-frequency noise that had $3 \mathrm{MHz}$ frequency was well attenuated by the low gain of the amplifier at the high frequencies, and the 
common-mode noise could be rejected by the high CMRR of the instrumentation amplifier. The conditioned output voltage of the GMR sensor matched pretty well with the high-bandwidth and high-accuracy current probe.

5. In addition to the GMR current sensor with a signal-conditioning circuit, the over-current protection circuit was designed using a comparator and gate driver. The designed over-current protection circuit showed a $240 \mathrm{~ns}$ delay to the turn of the gate driver, after over-current realization. The integrated gate driver circuit could drive both the high-side switch and the low-side switch, and the dead time between two signals could be controlled by an external resistor.

\subsection{Future Work}

1. Integrate the designed circuit on a real module.

2. The equations that derived from thermal experiment show the possibility of actively compensating the measurement error that results from varying temperature conditions. However, there are additional variables that can influence the temperature compensation loop. For example, the experiment was done with a constant current, whereas the real sensor will sense wide range of currents, and the derived equation cannot be guaranteed to be used with this condition. More experiments will be needed for more accurate modeling of the thermal model.

3. The location of the GMR sensor is missing a portion of low current from its linear range. By adjusting location of the sensor, the sensor can detect a wider range of current in a linear range.

4. The circuitry on the top DBC hinders true double-sided cooling. If possible, the gate driver circuit with over-current protection should be further simplified to have enough room for heat sinking. 


\section{REFERENCES}

[1] N. B. Nguyen, "Packaging Trends of Power Electronic Modules," presented at the Advancing microelectronics, 1997.

[2] J. G. Davids, "Power System Packaging Development," presented at the Advancing microelectronics, 1997.

[3] X. Chucheng, Z. Lingyin, T. Asada, W. G. Odendaal, and J. D. van Wyk, "An overview of integratable current sensor technologies," in Industry Applications Conference, 2003. 38th IAS Annual Meeting. Conference Record of the, 2003, pp. 1251-1258 vol.2.

[4] M. J. Caruso, T. Bratland, C. H. Smith, and R. Schneider, "A new perspective on magnetic-field sensing."

[5] R. P. Singh and A. M. Khambadkone, "Giant Magneto Resistive (GMR) Effect Based Current-sensing Technique for Low Voltage/High Current Voltage Regulator Modules," Power Electronics, IEEE Transactions on, vol. 23, pp. 915-925, 2008.

[6] E. R. Olson and R. D. Lorenz, "Integrating giant magnetoresistive current and thermal sensors in power electronic modules," in Applied Power Electronics Conference and Exposition, 2003. APEC '03. Eighteenth Annual IEEE, 2003, pp. 773-777 vol.2.

[7] A. Jander, R. S. Indeck, J. A. Brug, and J. H. Nickel, "A model for predicting heating of magnetoresistive heads," Magnetics, IEEE Transactions on, vol. 32, pp. 3392-3394, 1996.

[8] Y. Sungtaek Ju, X. Ren, W. Xiao, N. Smith, R. Fontana, L. Wen, K. Carey, H. Ho, D. Hsiao, and B. Gurney, "A combined experimental and numerical study of temperature rise in GMR sensors due to self-heating," Magnetics, IEEE Transactions on, vol. 37, pp. 1701-1703, 2001.

[9] N. Karrer and P. Hofer-Noser, "A new current measuring principle for power electronic applications," in Power Semiconductor Devices and ICs, 1999. ISPSD '99. Proceedings., The 11th International Symposium on, 1999, pp. 279-282.

[10] J. Daughton, J. Brown, E. Chen, R. Beech, A. Pohm, and W. Kude, "Magnetic-field sensors using GMR multilayer," Magnetics, IEEE Transactions on, vol. 30, pp. 4608-4610, 1994.

[11] X. Cao, G. Q. Lu, and K. D. T. Ngo, "Planar Power Module With Low Thermal Impedance and Low Thermomechanical Stress," Components, Packaging and Manufacturing Technology, IEEE Transactions on, vol. PP, pp. 1-1, 2012. 
[12] L. Dalessandro, N. Karrer, M. Ciappa, A. Castellazzi, and W. Fichtner, "Online and offline isolated current monitoring of parallel switched high-voltage multi-chip IGBT modules," in Power Electronics Specialists Conference, 2008. PESC 2008. IEEE, 2008, pp. 2600-2606.

[13] O. Elmatboly, A. Homaifar, and M. Zolghadri, "Giant magneto resistive sensing of critical power system parameters," in Industrial Electronics Society, 2005. IECON 2005. 31 st Annual Conference of IEEE, 2005, p. 6 pp.

[15] J. C. Jury, K. B. Klaassen, J. C. L. van Peppen, and S. X. Wang, "Measurement and analysis of noise sources in giant magnetoresistive sensors up to $6 \mathrm{GHz}$," Magnetics, IEEE Transactions on, vol. 38, pp. 3545-3555, 2002.

[16] E. R. Olson and R. D. Lorenz, "Effective Use of Miniature Multipoint Field-Based Current Sensors Without Magnetic Cores," Industry Applications, IEEE Transactions on, vol. 46, pp. 901-909, 2010.

[17] E. R. Oslon and R. D. Lorenz, "Integrated current-sensing for power electronic modules using GMR field detectors," in Power Electronics and Applications, 2005 European Conference on, 2005, pp. 9 pp.-P.9.

[18] P. R. Palmer, B. H. Stark, and J. C. Joyce, "Noninvasive measurement of chip currents in IGBT modules," in Power Electronics Specialists Conference, 1997. PESC '97 Record., 28th Annual IEEE, 1997, pp. 166-171 vol.1.

[19] W. Ranmuthu, K. T. M. Ranmuthu, A. V. Pohm, C. Kohl, C. S. Comstock, and M. Hassoun, "A Sensing Scheme For Giant Magneto-resistive Memories," in Magnetics Conference, 1993. INTERMAG '93., Digest of International, 1993, pp. DE-13-DE-13.

[20] P. Schneider, M. Horio, and R. Lorenz, "Integrating giant magneto-resistive (GMR) field detectors for high bandwidth current-sensing in power electronic modules," Industry Applications, IEEE Transactions on, vol. PP, pp. 1-1, 2012.

[21] H. N. Shah, Y. Xiao, T. P. Chow, R. J. Gutmann, E. R. Olson, S. H. Park, W. K. Lee, J. J. Connors, T. M. Jahns, and R. D. Lorenz, "Power electronics modules for inverter applications using flip-chip on flex-circuit technology," in Industry Applications Conference, 2004. 39th IAS Annual Meeting. Conference Record of the 2004 IEEE, 2004, pp. 1526-1533 vol.3.

[22] J. T. Strydom, J. D. van Wyk, C. K. Campbell, J. A. Ferreira, and M. F. K. Holm, "Packaging of an integrated planar power passive module for a power electronics converter: a $1 \mathrm{MHz}$ case study," Components and Packaging Technologies, IEEE Transactions on, vol. 25, pp. 260-265, 2002.

[23] N. A. Stutzke, S. E. Russek, D. P. Pappas, and M. Tondra, "Low-frequency noise measurements on commercial magnetoresistive magnetic-field sensors," Journal of Applied Physics, vol. 97, pp. 10Q107-10Q107-3, 2005. 
[24] L. Tong, N. Khai, G. Q. Lu, R. Burgos, F. Wang, and D. Boroyevich, "Comparison of current sharing among paralleled devices in wire-bonded and planar power modules," in Electric Ship Technologies Symposium, 2009. ESTS 2009. IEEE, 2009, pp. 406-411.

[25] L. Zhao, J. D. van Wyk, and W. G. Odendaal, "Planar embedded pick-up coil sensor for power electronic modules," in Applied Power Electronics Conference and Exposition, 2004. APEC '04. Nineteenth Annual IEEE, 2004, pp. 945-951 vol.2.

[26] J. A. Ferreira, W. A. Cronje, and W. A. Relihan, "Integration of high frequency current shunts in power electronic circuits," in Power Electronics Specialists Conference, 1992. PESC '92 Record., 23rd Annual IEEE, 1992, pp. 1284-1290 vol.2.

[27] A. R. A. M. Makky, H. Abo-Zied, F. N. Abdelbar, and P. Mutschler, "Design of the instrument current transformer for high frequency high power applications," in Power System Conference, 2008. MEPCON 2008. 12th International Middle-East, 2008, pp. 230-233.

[28] W. F. Ray and C. R. Hewson, "High performance Rogowski current transducers," in Industry Applications Conference, 2000. Conference Record of the 2000 IEEE, 2000, pp. 3083-3090 vol.5.

[29] K. Tan, T. Komakine, K. Yamakawa, Y. Kayano, H. Inoue, and M. Yamaguchi, "Detection of High-Frequency Magnetic-fields by a GMI Probe," Magnetics, IEEE Transactions on, vol. 42, pp. 3329-3331, 2006.

[30] E. R. Olson and R. D. Lorenz, "Using the dynamic behavior of superimposed fields for point field-based current-sensing," in Applied Power Electronics Conference and Exposition, 2006. APEC '06. Twenty-First Annual IEEE, 2006, p. 7 pp.

[31] L. Di Rienzo, R. Bazzocchi, and A. Manara, "Circular arrays of magnetic sensors for current measurement," Instrumentation and Measurement, IEEE Transactions on, vol. 50, pp. 1093-1096, 2001.

[32] Application note from NVE Corp. www.nve.com 


\section{List of Publications}

\section{CONFERENCE PAPERS}

[1] Woochan Kim and K.D.T. Ngo, "Integrated Current Sensor Using Giant Magneto

Resistive (GMR) for Planar Power Module" the 28th Annual IEEE Applied Power Electronics Conference \& Exposition, Long Beach, California, USA. 